\title{
Affinity for Scalar Fields to Dissipate
}

\author{
Arjun Berera ${ }^{*}$, Rudnei O. Ramos ${ }^{2,3 \dagger}$ \\ 1 Department of Physics and Astronomy, University of Edinburgh, \\ Edinburgh, EH9 3JZ, United Kingdom \\ 2 Department of Physics and Astronomy, Dartmouth College, \\ Hanover, New Hampshire 03755-3528, USA \\ and ${ }^{3}$ Departamento de Física Teórica, Instituto de Física, Universidade do Estado do Rio de Janeiro, \\ 20550-013 Rio de Janeiro, RJ, Brazil
}

\begin{abstract}
The zero temperature effective equation of motion is derived for a scalar field interacting with other fields. For a broad range of cases, involving interaction with as few as one or two fields, dissipative regimes are found for the scalar field system. The zero temperature limit constitutes a baseline effect that will be prevalent in any general statistical state. Thus, the results found here provide strong evidence that dissipation is the norm not exception for an interacting scalar field system. For application to inflationary cosmology, this provides convincing evidence that warm inflation could be a natural dynamics once proper treatment of interactions is done. The results found here also may have applicability to entropy production during the chiral phase transition in heavy ion collision.
\end{abstract}

PACS number(s): 98.80 Cq, 05.70.Ln, 11.10.Wx

\section{In Press Physical Review D (2001) hep-ph/0101049}

\section{INTRODUCTION}

Statistical mechanics generally expects an interacting system to equally distribute its energy amongst all the constituent degrees of freedom. Efforts to understand this most basic statement from quantum field theory have focused on simple dissipative systems. The most common example amongst these is the case of a quantum scalar field which is interacting with other fields and which has an amplitude that is initially displaced from equilibrium. There has been considerable interest in this kind of problem due to its many applications, ranging from the dynamics of condensates in condensed matter physics to the evolution of a inflaton field in inflationary cosmology (for a recent review see [1]). Several methods have been applied to this problem involving analytic and numerical analysis [2 14] as well as lattice simulations $[15,16]$.

Among the variety of dissipative dynamics that can be studied for such scalar field systems, the most tractable, given the present state of understanding in quantum statistical mechanics, is the adiabatic regime, where the motion of the scalar field amplitude is slow. One of the first implementation of this approximation was due to Caldeira and Leggett [17] for the problem of interacting quantum mechanical harmonic oscillators. Since then, generalizations of this kind of dynamics for the case of scalar fields have been implemented by several authors [8 12]. In particular, in an earlier work by us 12], a specific consistent solution regime for adiabatic dissipative dynamics was identified, in which the motion of the scalar field amplitude is overdamped. Our treatment in that work was restricted to the high-temperature regime. The purpose of this paper is to extend those results to zero temperature and to find valid parameter regions for the adiabatic dissipative regime. The key step is the inclusion of quasi-particles effects through the appropriate use of full two-point Green's functions at zero temperature. This plays a central role in the dynamics, as will be reviewed later in this paper.

A primary, but not singular, motivation for understanding the overdamped regime is to realize the warm inflation scenarios from first principles. The extension of the overdamped solution to zero temperature has important consequences for this goal. A careful examination of the earlier work [12] as well as 18,19] reveal that the high-temperature overdamped solutions came very close to a full realization of warm inflation from simple interacting models. Although ultimately what we found for that regime was that full warm inflation solutions only could be constructed for certain

\footnotetext{
${ }^{*}$ E-mail: ab@ph.ed.ac.uk; PPARC Advanced Fellow

${ }^{\dagger}$ E-mail: rudnei@peterpan.dartmouth.edu. ${ }^{3}$ Permanent address.
} 
complicated models [18 20], a foremost impediment to simpler solutions was the limitations of the high temperature approximation. The main accomplishment of this paper is to establish that the extension of the solution to $T=0$ can be adequately posed. Although only flat nonexpanding spacetime is treated in this paper, these results provide a necessary step towards the case of expanding spacetime. Furthermore, the results in this paper already are suggestive that once extension to expanding spacetime is done, warm inflation solution probably will be viable in most of the simple quantum field theory models.

The calculation in this paper is incomplete in that dissipation at zero temperature necessarily implies a nonequilibrium state, which immediately should be driven up to some excited statistical state. As such, the results presented here serve only as an indication for a nontrivial dissipative effect, which requires a more general nonequilibrium treatment for determining the precise nature of the excited statistical state. In this respect, the results in this paper are as yet insufficient for constructing completely consistent quantum field theory warm inflation solutions. Nevertheless, these calculations establish an important point, that interacting scalar field systems have an intrinsic and, in appropriate regimes, robust affinity to dissipate their energy. This fact in turn could place considerable doubt on a commonly followed, though unproven, assumption of inflationary cosmology, that dissipative effects of the scalar inflaton field can be ignored, thereby leading to a supercooled regime of inflation with vanishing, or negligible production of radiation.

Despite the unconventional implications our results suggest for inflationary cosmology, the argument for supercooling in scalar field driven inflation deserves a reexamination for the following reason. The basic statement of supercooled inflation is that a single degree of freedom, the zero mode of the inflaton, maintains all the energy of the universe during the entire duration of inflation in the form of potential, or equivalently vacuum, energy. However, the curious point is, even if this single degree were to allow a minuscule fraction of the energy to be released, say one part in $10^{20}$, it still would constitute a significant radiation energy density component in the universe. For example, for inflation with vacuum energy at the GUT scale $\sim 10^{15-16} \mathrm{GeV}$, leaking one part in $10^{20}$ of this energy into radiation corresponds to a temperature of $10^{11} \mathrm{GeV}$, which is nonnegligible. In fact, the most relevant lower bound that cosmology places on the temperature after inflation comes from the success of hot Big-Bang nucleosynthesis, which thus requires the universe to be within the radiation dominated regime by $T \gtrsim 1 \mathrm{GeV}$. This limit can be met in the above example by dissipating as little as one part in $10^{60}$ of the vacuum energy into radiation. Thus, from the perspective of both interacting field theory and basic notions of equipartition, it appears to be a highly tuned requirement of supercooled inflation to prohibit the inflaton from even such tiny amounts of dissipation. On the flip side, the warm inflation picture demonstrates [21] that by relaxing this requirement, the most unnatural and technically intractable aspect of supercooled inflation, reheating, becomes unnecessary. As such, despite the technical complications in computing dissipative effects, as exemplified by this and the earlier papers, on general grounds their presence should be a natural expectation.

The paper is organized as follows. Our real-time dissipative formalism is reviewed in Sec. II. Explicit expressions are given here for the real-time, fully dressed Bose and Fermi two-point Green's functions, which are valid in the entire temperature range, including $T=0$. This formalism then is applied in Sects. II and III to obtain the effective equation of motion of the system, here a scalar field that has a classical amplitude out of equilibrium at $T=0$, which is coupled to fields that act as a reservoir bath for dissipative energy exchange. Two types of models are treated in Sections II and III, which we have denoted respectively as direct decay models, where the system field directly decays to light particles that are part of the environment, and indirect decay models, where this process is mediated by intermediate fields. In Sec. IV, we apply the effective equations of motion to estimate the magnitude of radiation energy production. Finally in Sec. V, we give concluding remarks and comment on the implication of our results for the case of an expanding spacetime, which is relevant for inflationary cosmology. An Appendix is also included in which the computations are given of the zero temperature decay widths that are used in the paper.

\section{DIRECT DECAY MODELS}

In this section, models will be studied where the system, here a scalar field $\Phi$, is coupled to bath fields of lighter mass than itself, thus allowing $\Phi$ to directly decay into the bath fields. In the first model the scalar field interacts with a set of $N_{\psi}$ fermion fields with Lagrangian density

$$
\mathcal{L}\left[\Phi, \bar{\psi}_{k}, \psi_{k}\right]=\frac{1}{2}\left(\partial_{\mu} \Phi\right)^{2}-\frac{m_{\phi}^{2}}{2} \Phi^{2}-\frac{\lambda}{4 !} \Phi^{4}+\sum_{k=1}^{N_{\psi}} \bar{\psi}_{k}\left[i \not \partial-m_{\psi_{k}}-h_{k} \Phi\right] \psi_{k}
$$

The $\Phi$ field is decomposed into a classical background component $\varphi$ and a quantum fluctuation part as $\Phi=\varphi+\phi$, with

$$
\langle\Phi\rangle=\varphi,\langle\phi\rangle=0
$$


and where the background component is assumed to be homogeneous $\varphi \equiv \varphi(t)$. Using the tadpole method", where we impose $\langle\phi\rangle=0$ at all orders in perturbation theory, it leads to the condition that the sum of all tadpole terms vanish. The effective equation of motion (EOM) for $\varphi(t)$ then becomes

$$
\ddot{\varphi}(t)+m_{\phi}^{2} \varphi(t)+\frac{\lambda}{6} \varphi^{3}(t)+\frac{\lambda}{2} \varphi(t)\left\langle\phi^{2}\right\rangle+\frac{\lambda}{6}\left\langle\phi^{3}\right\rangle+\sum_{k=1}^{N_{\psi}} h_{k}\left\langle\overline{\psi_{k}} \psi_{k}\right\rangle=0,
$$

where $\left\langle\phi^{2}\right\rangle,\left\langle\phi^{3}\right\rangle$ and $\left\langle\bar{\psi}_{k} \psi_{k}\right\rangle$ are given in terms of the coincidence limit of the (causal) two-point Green's functions $G_{\phi}^{++}\left(x, x^{\prime}\right)$ and $S_{\psi}^{++}\left(x, x^{\prime}\right)$. These Green's functions are obtained from the $(1,1)$-component of the real time matrix of full propagators which satisfy the appropriate Schwinger-Dyson equations (see, e.g., 10,12] for further details)

$$
\left[\square+m_{\phi}^{2}+\frac{\lambda}{2} \varphi^{2}\right] G_{\phi}\left(x, x^{\prime}\right)+\int d^{4} z \Sigma_{\phi}(x, z) G_{\phi}\left(z, x^{\prime}\right)=i \delta\left(x, x^{\prime}\right)
$$

and

$$
\left[i \not \partial-m_{\psi_{k}}-h_{k} \varphi\right] S_{\psi_{k}}\left(x, x^{\prime}\right)+\int d^{4} z \Sigma_{\psi_{k}}(x, z) S_{\psi_{k}}\left(z, x^{\prime}\right)=i \delta\left(x, x^{\prime}\right) .
$$

The momentum-space Fourier transform of $G_{\phi}\left(x, x^{\prime}\right)$ (for the scalar field) can be expressed in the form

$$
G_{\phi}\left(x, x^{\prime}\right)=i \int \frac{d^{3} q}{(2 \pi)^{3}} e^{i \mathbf{q} \cdot\left(\mathbf{x}-\mathbf{x}^{\prime}\right)}\left(\begin{array}{ll}
G_{\phi}^{++}\left(\mathbf{q}, t-t^{\prime}\right) & G_{\phi}^{+-}\left(\mathbf{q}, t-t^{\prime}\right) \\
G_{\phi}^{-+}\left(\mathbf{q}, t-t^{\prime}\right) & G_{\phi}^{--}\left(\mathbf{q}, t-t^{\prime}\right)
\end{array}\right),
$$

where

$$
\begin{aligned}
& G_{\phi}^{++}\left(\mathbf{q}, t-t^{\prime}\right)=G_{\phi}^{>}\left(\mathbf{q}, t-t^{\prime}\right) \theta\left(t-t^{\prime}\right)+G_{\phi}^{<}\left(\mathbf{q}, t-t^{\prime}\right) \theta\left(t^{\prime}-t\right) \\
& G_{\phi}^{--}\left(\mathbf{q}, t-t^{\prime}\right)=G_{\phi}^{>}\left(\mathbf{q}, t-t^{\prime}\right) \theta\left(t^{\prime}-t\right)+G_{\phi}^{<}\left(\mathbf{q}, t-t^{\prime}\right) \theta\left(t-t^{\prime}\right), \\
& G_{\phi}^{+-}\left(\mathbf{q}, t-t^{\prime}\right)=G_{\phi}^{<}\left(\mathbf{q}, t-t^{\prime}\right), \\
& G_{\phi}^{-+}\left(\mathbf{q}, t-t^{\prime}\right)=G_{\phi}^{>}\left(\mathbf{q}, t-t^{\prime}\right) .
\end{aligned}
$$

In these expressions the fully dressed (field independent) two-point functions, at finite temperature $T=1 / \beta$, under the approximation that the spectral function for the scalar field has the standard Breit-Wigner form, are given by

$$
\begin{aligned}
G_{\phi}^{>}\left(\mathbf{q}, t-t^{\prime}\right) & =\frac{1}{2 \omega_{\phi}}\left\{\left[1+n_{\phi}\left(\omega_{\phi}-i \Gamma_{\phi}\right)\right] e^{-i\left(\omega_{\phi}-i \Gamma_{\phi}\right)\left(t-t^{\prime}\right)}+n_{\phi}\left(\omega_{\phi}+i \Gamma_{\phi}\right) e^{i\left(\omega_{\phi}+i \Gamma_{\phi}\right)\left(t-t^{\prime}\right)}\right\} \theta\left(t-t^{\prime}\right) \\
& +\frac{1}{2 \omega_{\phi}}\left\{\left[1+n_{\phi}\left(\omega_{\phi}+i \Gamma_{\phi}\right)\right] e^{-i\left(\omega_{\phi}+i \Gamma_{\phi}\right)\left(t-t^{\prime}\right)}+n_{\phi}\left(\omega_{\phi}-i \Gamma_{\phi}\right) e^{i\left(\omega_{\phi}-i \Gamma_{\phi}\right)\left(t-t^{\prime}\right)}\right\} \theta\left(t^{\prime}-t\right), \\
G_{\phi}^{<}\left(\mathbf{q}, t-t^{\prime}\right) & =G_{\phi}^{>}\left(\mathbf{q}, t^{\prime}-t\right),
\end{aligned}
$$

where $n_{\phi}$ is the Bose distribution function, $\omega_{\phi} \equiv \omega_{\phi}(\mathbf{q})$ is the particle's dispersion relation and $\Gamma_{\phi}$ is the $\phi$ decay width, defined as usual in terms of the field self-energy by

$$
\Gamma_{\phi}(q)=\frac{\operatorname{Im} \Sigma_{\phi}\left(\mathbf{q}, \omega_{\phi}\right)}{2 \omega_{\phi}}
$$

For the fermion fields we have instead that

$$
\begin{aligned}
& S_{\psi}^{++}\left(\mathbf{q}, t-t^{\prime}\right)=S_{\psi}^{>}\left(\mathbf{q}, t-t^{\prime}\right) \theta\left(t-t^{\prime}\right)+S_{\psi}^{<}\left(\mathbf{q}, t-t^{\prime}\right) \theta\left(t^{\prime}-t\right), \\
& S_{\psi}^{--}\left(\mathbf{k}, t-t^{\prime}\right)=S_{\psi}^{>}\left(\mathbf{q}, t-t^{\prime}\right) \theta\left(t^{\prime}-t\right)+S_{\psi}^{<}\left(\mathbf{q}, t-t^{\prime}\right) \theta\left(t-t^{\prime}\right), \\
& S_{\psi}^{+-}\left(\mathbf{q}, t-t^{\prime}\right)=S_{\psi}^{<}\left(\mathbf{q}, t-t^{\prime}\right), \\
& S_{\psi}^{-+}\left(\mathbf{q}, t-t^{\prime}\right)=S_{\psi}^{>}\left(\mathbf{q}, t-t^{\prime}\right) .
\end{aligned}
$$

\footnotetext{
${ }^{1}$ For earlier references on the tadpole method applied to determining the equation of motion of a scalar field in the real time formalism, see for instance Ref. 22.

${ }^{2}$ We thank Ian Lawrie for pointing out the correct form of these equations.
} 
In this case $S_{\psi}^{>,<}$are given byß

$$
\begin{aligned}
& S_{\psi}^{>}\left(\mathbf{q}, t-t^{\prime}\right)=-\frac{1}{2 \omega_{\psi}}\left\{e^{-i\left(\omega_{\psi}-i \Gamma_{\psi}\right)\left(t-t^{\prime}\right)}\left[\left(\omega_{\psi}-i \Gamma_{\psi}\right) \gamma_{0}-\not \mathbf{q}+m_{\psi, r}-i \frac{\Gamma_{\psi}\left(\omega_{\psi}-i \Gamma_{\psi}\right)}{m_{\psi, r}}\right]\right. \\
& \times\left[1-n_{\psi}\left(\omega_{\psi}-i \Gamma_{\psi}\right)\right] \\
& \left.-e^{i\left(\omega_{\psi}+i \Gamma_{\psi}\right)\left(t-t^{\prime}\right)}\left[-\left(\omega_{\psi}+i \Gamma_{\psi}\right) \gamma_{0}-\not{q}+m_{\psi, r}+i \frac{\Gamma_{\psi}\left(\omega_{\psi}+i \Gamma_{\psi}\right)}{m_{\psi, r}}\right] n_{\psi}\left(\omega_{\psi}+i \Gamma_{\psi}\right)\right\} \theta\left(t-t^{\prime}\right) \\
& -\frac{1}{2 \omega_{\psi}}\left\{e^{-i\left(\omega_{\psi}+i \Gamma_{\psi}\right)\left(t-t^{\prime}\right)}\left[\left(\omega_{\psi}+i \Gamma_{\psi}\right) \gamma_{0}-\not{q}+m_{\psi, r}+i \frac{\Gamma_{\psi}\left(\omega_{\psi}+i \Gamma_{\psi}\right)}{m_{\psi, r}}\right]\right. \\
& \times\left[1-n_{\psi}\left(\omega_{\psi}+i \Gamma_{\psi}\right)\right] \\
& \left.-e^{i\left(\omega_{\psi}-i \Gamma_{\psi}\right)\left(t-t^{\prime}\right)}\left[-\left(\omega_{\psi}-i \Gamma_{\psi}\right) \gamma_{0}-\not{q}+m_{\psi, r}-i \frac{\Gamma_{\psi}\left(\omega_{\psi}-i \Gamma_{\psi}\right)}{m_{\psi, r}}\right] n_{\psi}\left(\omega_{\psi}-i \Gamma_{\psi}\right)\right\} \theta\left(t^{\prime}-t\right),
\end{aligned}
$$

and

$$
\begin{aligned}
& S_{\psi}^{<}\left(\mathbf{q}, t-t^{\prime}\right)=\frac{1}{2 \omega_{\psi}}\left\{e^{i\left(\omega_{\psi}+i \Gamma_{\psi}\right)\left(t-t^{\prime}\right)}\left[-\left(\omega_{\psi}+i \Gamma_{\psi}\right) \gamma_{0}-\not \mathbf{q}+m_{\psi, r}+i \frac{\Gamma_{\psi}\left(\omega_{\psi}+i \Gamma_{\psi}\right)}{m_{\psi, r}}\right]\right. \\
& \times\left[1-n_{\psi}\left(\omega_{\psi}+i \Gamma_{\psi}\right)\right] \\
& \left.-e^{-i\left(\omega_{\psi}-i \Gamma_{\psi}\right)\left(t-t^{\prime}\right)}\left[\left(\omega_{\psi}-i \Gamma_{\psi}\right) \gamma_{0}-\not \mathbf{q}+m_{\psi, r}-i \frac{\Gamma_{\psi}\left(\omega_{\psi}-i \Gamma_{\psi}\right)}{m_{\psi, r}}\right] n_{\psi}\left(\omega_{\psi}-i \Gamma_{\psi}\right)\right\} \theta\left(t-t^{\prime}\right) \\
& +\frac{1}{2 \omega_{\psi}}\left\{e^{i\left(\omega_{\psi}-i \Gamma_{\psi}\right)\left(t-t^{\prime}\right)}\left[-\left(\omega_{\psi}-i \Gamma_{\psi}\right) \gamma_{0}-\not \mathbf{q}+m_{\psi, r}-i \frac{\Gamma_{\psi}\left(\omega_{\psi}-i \Gamma_{\psi}\right)}{m_{\psi, r}}\right]\right. \\
& \times\left[1-n_{\psi}\left(\omega_{\psi}-i \Gamma_{\psi}\right)\right] \\
& \left.-e^{-i\left(\omega_{\psi}+i \Gamma_{\psi}\right)\left(t-t^{\prime}\right)}\left[\left(\omega_{\psi}+i \Gamma_{\psi}\right) \gamma_{0}-\not \mathbf{q}+m_{\psi, r}+i \frac{\Gamma_{\psi}\left(\omega_{\psi}+i \Gamma_{\psi}\right)}{m_{\psi, r}}\right] n_{\psi}\left(\omega_{\psi}+i \Gamma_{\psi}\right)\right\} \theta\left(t^{\prime}-t\right),
\end{aligned}
$$

where $n_{\psi}=1 /\left(e^{\beta \omega_{\psi}}+1\right), \omega_{\psi}=\sqrt{\mathbf{q}^{2}+m_{\psi, r}^{2}}$, with $m_{\psi, r}=m_{\psi}+\operatorname{Re} \Sigma_{\psi}$ and

$$
\Gamma_{\psi}(q)=\operatorname{Im} \Sigma_{\psi}\left(\omega_{\psi}, \mathbf{q}\right) \frac{m_{\psi}}{\omega_{\psi}} .
$$

In this paper we will be interested in studying dissipation only of the background scalar field $\varphi$ at $T=0$. Therefore we will restrict ourselves just to the zero temperature expressions of the above equations.

It will be assumed the couplings $\lambda, h_{k} \ll 1$, so that perturbation theory can be consistently formulated with subleading terms neglected. Then by perturbatively expanding the field averages in Eq. (2.2), up to two-loop order in the scalar loops (higher order loop terms involving fermions also are higher order in the perturbation expansion, as we will discuss later), the effective $\mathrm{EOM}$ for $\varphi(t)$ is

$$
\begin{aligned}
& \ddot{\varphi}(t)+\left(m_{\phi}^{2}+\frac{\lambda}{2}\left\langle\phi^{2}\right\rangle_{0}\right) \varphi(t)+\frac{\lambda}{6} \varphi^{3}(t) \\
& +\lambda \varphi(t) \int_{-\infty}^{t} d t^{\prime} \frac{\lambda}{2} \varphi^{2}\left(t^{\prime}\right) \int \frac{d^{3} \mathbf{q}}{(2 \pi)^{3}} \operatorname{Im}\left[G_{\phi}^{++}\left(\mathbf{q}, t-t^{\prime}\right)\right]^{2} \\
& +\frac{\lambda^{2}}{3} \int_{-\infty}^{t} d t^{\prime} \varphi\left(t^{\prime}\right) \int \frac{d^{3} \mathbf{q}_{1}}{(2 \pi)^{3}} \frac{d^{3} \mathbf{q}_{2}}{(2 \pi)^{3}} \operatorname{Im}\left[G_{\phi}^{++}\left(\mathbf{q}_{1}, t-t^{\prime}\right) G_{\phi}^{++}\left(\mathbf{q}_{2}, t-t^{\prime}\right) G_{\phi}^{++}\left(\mathbf{q}_{1}+\mathbf{q}_{2}, t-t^{\prime}\right)\right] \\
& -4 \sum_{k=1}^{N_{\psi}} h_{k, \phi} \int_{-\infty}^{t} d t^{\prime} h_{k} \varphi\left(t^{\prime}\right) \int \frac{d^{3} q}{(2 \pi)^{3}} \operatorname{Im}\left[S_{\alpha \beta}^{++}\left(\mathbf{q}, t-t^{\prime}\right) S^{++\beta \alpha}\left(\mathbf{q}, t^{\prime}-t\right)\right]=0,
\end{aligned}
$$

with $\langle\ldots\rangle_{0}$ meaning the vacuum expectation value at zero background field $\varphi=0$. Using the explicit expressions for the Green's functions in Eq. (2.13) and at $T=0$, this EOM becomes

\footnotetext{
${ }^{3}$ Analogous expressions for the general nonequilibrium case are given in 23 .
} 


$$
\begin{aligned}
& \ddot{\varphi}(t)+\left(m_{\phi}^{2}+\frac{\lambda}{2}\left\langle\phi^{2}\right\rangle_{0}\right) \varphi(t)+\frac{\lambda}{6} \varphi^{3}(t)-\lambda^{2} \varphi(t) \int_{-\infty}^{t} d t^{\prime} \varphi^{2}\left(t^{\prime}\right) \int \frac{d^{3} q}{(2 \pi)^{3}} \frac{\sin \left(2 \omega_{\phi}\left|t-t^{\prime}\right|\right)}{8 \omega_{\phi}^{2}} e^{-2 \Gamma_{\phi}(q)\left|t-t^{\prime}\right|} \\
& -\frac{\lambda^{2}}{3} \int_{-\infty}^{t} d t^{\prime} \varphi\left(t^{\prime}\right) \int \frac{d^{3} \mathbf{q}_{1}}{(2 \pi)^{3}} \frac{d^{3} \mathbf{q}_{2}}{(2 \pi)^{3}} \frac{\sin \left[\left(\omega_{\phi}\left(\mathbf{q}_{1}\right)+\omega_{\phi}\left(\mathbf{q}_{2}\right)+\omega_{\phi}\left(\mathbf{q}_{1}+\mathbf{q}_{2}\right)\right)\left|t-t^{\prime}\right|\right]}{8 \omega_{\phi}\left(\mathbf{q}_{1}\right) \omega_{\phi}\left(\mathbf{q}_{2}\right) \omega_{\phi}\left(\mathbf{q}_{1}+\mathbf{q}_{2}\right)} \\
& \times e^{-\left[\Gamma_{\phi}\left(\mathbf{q}_{1}\right)+\Gamma_{\phi}\left(\mathbf{q}_{2}\right)+\Gamma_{\phi}\left(\mathbf{q}_{1}+\mathbf{q}_{2}\right)\right]\left|t-t^{\prime}\right|} \\
& -2 \sum_{k=1}^{N_{\psi}} h_{k}^{2} \int_{-\infty}^{t} d t^{\prime} \varphi\left(t^{\prime}\right) \int \frac{d^{3} q}{(2 \pi)^{3}} \frac{e^{-2 \Gamma_{\psi_{k}}(q)\left|t-t^{\prime}\right|}}{\omega_{\psi_{k}}^{2} m_{\psi, r}^{2}}\left\{2 \Gamma_{\psi_{k}}^{3} \omega_{\psi_{k}} \cos \left(2 \omega_{\psi_{k}}\left|t-t^{\prime}\right|\right)\right. \\
& \left.+\left[2 m_{\psi, r}^{2}\left(\omega_{\psi_{k}}^{2}-m_{\psi_{k}}^{2}\right)+\Gamma_{\psi_{k}}^{2}\left(\omega_{\psi_{k}}^{2}+m_{\psi, r}^{2}-\Gamma_{\psi_{k}}\right)\right] \sin \left(2 \omega_{\psi_{k}}\left|t-t^{\prime}\right|\right)\right\}=0 .
\end{aligned}
$$

The temporally nonlocal terms of the types appearing in the above equation have been shown in 2, 2, 11, 14, 24, 25, to lead to dissipative dynamics in the EOMs. This can be made more explicit by an appropriate integration by parts in the time integrals in Eq. (2.14) (see 225]) to obtain the result (at $T=0$ )

$$
\begin{aligned}
& \ddot{\varphi}(t)+\bar{m}_{\phi}^{2} \varphi(t)+\frac{\bar{\lambda}}{6} \varphi^{3}(t) \\
& +\lambda^{2} \varphi(t) \int_{-\infty}^{t} d t^{\prime} \varphi\left(t^{\prime}\right) \dot{\varphi}\left(t^{\prime}\right) \int \frac{d^{3} q}{(2 \pi)^{3}} \frac{\left[\omega_{\phi} \cos \left(2 \omega_{\phi}\left|t-t^{\prime}\right|\right)+\Gamma_{\phi} \sin \left(2 \omega_{\phi}\left|t-t^{\prime}\right|\right)\right]}{8 \omega_{\phi}^{2}\left(\Gamma_{\phi}^{2}+\omega_{\phi}^{2}\right)} e^{-2 \Gamma_{\phi}(q)\left|t-t^{\prime}\right|} \\
& +\frac{\lambda^{2}}{3} \int_{-\infty}^{t} d t^{\prime} \dot{\varphi}\left(t^{\prime}\right) \int \frac{d^{3} \mathbf{q}_{1}}{(2 \pi)^{3}} \frac{d^{3} \mathbf{q}_{2}}{(2 \pi)^{3}}\left\{\left[\omega_{\phi}\left(\mathbf{q}_{1}\right)+\omega_{\phi}\left(\mathbf{q}_{2}\right)+\omega_{\phi}\left(\mathbf{q}_{1}+\mathbf{q}_{2}\right)\right]\right. \\
& \times \cos \left[\left(\omega_{\phi}\left(\mathbf{q}_{1}\right)+\omega_{\phi}\left(\mathbf{q}_{2}\right)+\omega_{\phi}\left(\mathbf{q}_{1}+\mathbf{q}_{2}\right)\right)\left|t-t^{\prime}\right|\right] \\
& \left.+\left[\Gamma_{\phi}\left(\mathbf{q}_{1}\right)+\Gamma_{\phi}\left(\mathbf{q}_{2}\right)+\Gamma_{\phi}\left(\mathbf{q}_{1}+\mathbf{q}_{2}\right)\right] \sin \left[\left(\omega_{\phi}\left(\mathbf{q}_{1}\right)+\omega_{\phi}\left(\mathbf{q}_{2}\right)+\omega_{\phi}\left(\mathbf{q}_{1}+\mathbf{q}_{2}\right)\right)\left|t-t^{\prime}\right|\right]\right\} \\
& \times \frac{e^{-\left[\Gamma_{\phi}\left(\mathbf{q}_{1}\right)+\Gamma_{\phi}\left(\mathbf{q}_{2}\right)+\Gamma_{\phi}\left(\mathbf{q}_{1}+\mathbf{q}_{2}\right)\right]\left|t-t^{\prime}\right|}}{8 \omega_{\phi}\left(\mathbf{q}_{1}\right) \omega_{\phi}\left(\mathbf{q}_{2}\right) \omega_{\phi}\left(\mathbf{q}_{1}+\mathbf{q}_{2}\right)} \\
& \times \frac{1}{\left[\Gamma_{\phi}\left(\mathbf{q}_{1}\right)+\Gamma_{\phi}\left(\mathbf{q}_{2}\right)+\Gamma_{\phi}\left(\mathbf{q}_{1}+\mathbf{q}_{2}\right)\right]^{2}+\left[\omega_{\phi}\left(\mathbf{q}_{1}\right)+\omega_{\phi}\left(\mathbf{q}_{2}\right)+\omega_{\phi}\left(\mathbf{q}_{1}+\mathbf{q}_{2}\right)\right]^{2}} \\
& +\sum_{k=1}^{N_{\psi}} h_{k}^{2} \int_{-\infty}^{t} d t^{\prime} \dot{\varphi}\left(t^{\prime}\right) \int \frac{d^{3} q}{(2 \pi)^{3}} \frac{e^{-2 \Gamma_{\psi_{k}}(q)\left|t-t^{\prime}\right|}}{\omega_{\psi_{k}}^{2} m_{\psi, r}^{2}\left(\Gamma_{\psi_{k}}^{2}+\omega_{\psi_{k}}^{2}\right)} \\
& \times\left\{\left[\Gamma_{\psi_{k}}^{4} \omega_{\psi_{k}}+2 \omega_{\psi_{k}}^{3} m_{\psi, r}^{2}-2 m_{\psi, r}^{4} \omega_{\psi_{k}}+\Gamma_{\psi_{k}}^{2} \omega_{\psi_{k}}\left(\omega_{\psi_{k}}^{2}+m_{\psi, r}^{2}\right)\right] \cos \left(2 \omega_{\psi_{k}}\left|t-t^{\prime}\right|\right)\right. \\
& \left.+\left[\Gamma_{\psi_{k}}\left(2 m_{\psi_{k}}^{2}-\Gamma_{\psi_{k}}^{2}\right)\left(\omega_{\psi_{k}}^{2}-m_{\psi, r}^{2}\right)-\Gamma_{\psi_{k}}^{5}\right] \sin \left(2 \omega_{\psi_{k}}\left|t-t^{\prime}\right|\right)\right\}=0
\end{aligned}
$$

where $\bar{m}_{\phi}$ and $\bar{\lambda}$ are the effective mass and coupling constant given respectively by

$$
\begin{aligned}
\bar{m}_{\phi}^{2} & =m_{\phi}^{2}+\lambda \int \frac{d^{3} q}{(2 \pi)^{3}} \frac{1}{4 \omega_{\phi}}+\sum_{k=1}^{N_{\psi}} h_{k}^{2} \int \frac{d^{3} q}{(2 \pi)^{3}}\left[2 \frac{m_{\psi, r}^{2}-\omega_{\psi_{k}}^{2}}{\omega_{\psi_{k}}^{3}}+\mathcal{O}\left(\frac{\Gamma_{\psi_{k}}^{2}}{\omega_{\psi_{k}}^{2}}\right)\right] \\
& -\frac{\lambda^{2}}{3} \int \frac{d^{3} \mathbf{q}_{1}}{(2 \pi)^{3}} \frac{d^{3} \mathbf{q}_{2}}{(2 \pi)^{3}}\left\{\frac{1}{8 \omega_{\phi}\left(\mathbf{q}_{1}\right) \omega_{\phi}\left(\mathbf{q}_{2}\right) \omega_{\phi}\left(\mathbf{q}_{1}+\mathbf{q}_{2}\right)\left[\omega_{\phi}\left(\mathbf{q}_{1}\right)+\omega_{\phi}\left(\mathbf{q}_{2}\right)+\omega_{\phi}\left(\mathbf{q}_{1}+\mathbf{q}_{2}\right)\right]}\right. \\
& \left.+\mathcal{O}\left(\frac{\Gamma_{\phi}^{2}}{\omega_{\phi}^{2}}\right)\right\}
\end{aligned}
$$

and

$$
\bar{\lambda}=\lambda-\lambda^{2} \int \frac{d^{3} q}{(2 \pi)^{3}}\left[\frac{1}{16 \omega_{\phi}^{3}}+\mathcal{O}\left(\frac{\Gamma_{\phi}^{2}}{\omega_{\phi}^{2}}\right)\right] .
$$

Both $\bar{m}_{\phi}$ and $\bar{\lambda}$ naively appear divergent in Eqs. (2.16) and (2.17) respectively, due to the perturbative correction terms. However, they are rendered finite by the usual introduction of counterterms to renormalize the mass and coupling constant in the original Lagrangian [11. In this procedure, the bare (infinite) mass and scalar self-coupling in the Lagrangian are $m=m_{\phi, r}+\delta m_{\phi}$ and $\lambda=\lambda_{r}+\delta \lambda$, where the counterterms $\delta m_{\phi}$ and $\delta \lambda$ cancel the divergent 
contributions in Eqs. (2.16) and (2.17) in the usual way. In what follows, we then can just interpret the masses and couplings appearing in our equations as the renormalized ones and we will omit any additional subscript and overbars for simplicity, so hereafter $\bar{m} \rightarrow m$ and $\bar{\lambda} \rightarrow \lambda$.

\section{A. The EOM in the adiabatic-Markovian approximation}

The effective equation of motion Eq. (2.15) is the main result of this section. As should appear evident, this equation is difficult to solve either analytically or numerically, since memory of the past history of $\varphi$ is required at each stage of evolution. Falling short of a detailed analysis of this equation in this paper, we would like some rough estimates of the dissipative effects described by the equation. In particular, as a first approximation to Eq. (2.15), we will explore the derivative expansion at leading order for the temporally nonlocal terms. This amounts to substituting $t^{\prime} \rightarrow t$ in the arguments of the fields entering in the time integrals. This is equivalent to a Markovian approximation for the dissipative kernels in Eq. 2.15). A solution regime for $\varphi$ where such an approximation might be valid is the adiabatic regime, where the motion of $\varphi$ is slow. In this and the next subsections, we will establish a self-consistent solution regime composed of both the adiabatic and Markovian approximations. To proceed, first the Markovian approximation will be implemented and then self-consistent solution regimes will be identified. Thus upon implementing the Markovian approximation, we then can easily perform the $t^{\prime}$ integrals and obtain

$$
\begin{aligned}
& \ddot{\varphi}(t)+m_{\phi}^{2} \varphi(t)+\frac{\lambda}{6} \varphi^{3}(t)+\lambda^{2} \varphi^{2}(t) \dot{\varphi}(t) \int \frac{d^{3} q}{(2 \pi)^{3}} \frac{\Gamma_{\phi}}{8 \omega_{\phi}\left(\omega_{\phi}^{2}+\Gamma_{\phi}^{2}\right)^{2}} \\
& +\frac{\lambda^{2}}{3} \dot{\varphi}(t) \int \frac{d^{3} \mathbf{q}_{1}}{(2 \pi)^{3}} \frac{d^{3} \mathbf{q}_{2}}{(2 \pi)^{3}} \frac{\left[\Gamma_{\phi}\left(\mathbf{q}_{1}\right)+\Gamma_{\phi}\left(\mathbf{q}_{2}\right)+\Gamma_{\phi}\left(\mathbf{q}_{1}+\mathbf{q}_{2}\right)\right]\left[\omega_{\phi}\left(\mathbf{q}_{1}\right)+\omega_{\phi}\left(\mathbf{q}_{2}\right)+\omega_{\phi}\left(\mathbf{q}_{1}+\mathbf{q}_{2}\right)\right]}{4 \omega_{\phi}\left(\mathbf{q}_{1}\right) \omega_{\phi}\left(\mathbf{q}_{2}\right) \omega_{\phi}\left(\mathbf{q}_{1}+\mathbf{q}_{2}\right)} \\
& \times \frac{1}{\left\{\left[\Gamma_{\phi}\left(\mathbf{q}_{1}\right)+\Gamma_{\phi}\left(\mathbf{q}_{2}\right)+\Gamma_{\phi}\left(\mathbf{q}_{1}+\mathbf{q}_{2}\right)\right]^{2}+\left[\omega_{\phi}\left(\mathbf{q}_{1}\right)+\omega_{\phi}\left(\mathbf{q}_{2}\right)+\omega_{\phi}\left(\mathbf{q}_{1}+\mathbf{q}_{2}\right)\right]^{2}\right\}^{2}} \\
& +\sum_{k=1}^{N_{\psi}} h_{k}^{2} \dot{\varphi}(t) \int \frac{d^{3} q}{(2 \pi)^{3}} \frac{\left(\Gamma_{\psi_{k}}^{2}+2 \omega_{\psi_{k}}^{2}-2 m_{\psi_{k}}^{2}\right)}{\omega_{\psi_{k}}\left(\Gamma_{\psi_{k}}^{2}+\omega_{\psi_{k}}^{2}\right)^{2}} \Gamma_{\psi_{k}}=0 .
\end{aligned}
$$

An additional simplification can be achieved by demanding that the (effective) field masses satisfy the inequality $m_{\phi}>2 m_{\psi_{k}}$. In this case, at zero temperature, the only contribution to the decay widths in Eqs. (2.8) and (2.12) come from the imaginary part of the one loop contribution to the self-energy for $\Phi$. This is given by the internal fermionic propagators diagram, which then represents the decay rate for the kinematically allowed process $\Phi \rightarrow \psi_{k}+\bar{\psi}_{k}$. We therefore have that $\Gamma_{\psi_{k}}=0$ and $\Gamma_{\phi}$ is given by (see the Appendix)

$$
\Gamma_{\phi}(q)=\sum_{k=1}^{N_{\psi}} \frac{h_{k}^{2}}{8 \pi \omega_{\phi}(\mathbf{q})} m_{\phi}^{2}\left(1-\frac{4 m_{\psi_{k}}^{2}}{m_{\phi}^{2}}\right)^{\frac{3}{2}}
$$

We must point out that even though in this case the contribution to the dissipative term in Eq. 2.18) coming from the fermionic loop vanishes, i.e. $\Gamma_{\psi_{k}}=0$, this is a consequence of assuming the Markovian approximation for the dissipative kernels. In the general case, the last term in the non-local EOM, Eq. (2.15), with $\Gamma_{\psi_{k}}=0$, still can be interpreted as a dissipative contribution [2,13,14, to the EOM. Therefore, here the Markovian approximation somewhat under-estimates the whole dissipative nature of the non-local kernels in (2.15). We expect this not to invalidate our main objective here, which is to determine the viability of adiabatic dissipative behavior in the background field evolution and the intrinsic dissipative nature of the field dynamics.

As an aside, it is interesting to discuss briefly the interpretation of dissipation coming from the second non-local dissipative term in Eq. (2.15) when $\Gamma_{\psi}=0$. Though for this case, particle decay does not contribute to dissipation, there still is dissipation due to "off-shell" excitation of virtual states which leads to decoherence and power law decay of the background field amplitude [2], in the absence of the scalar field self-interactions. This contrasts with the first dissipative term in Eq. (2.15), in which dissipation truly is coming from the real scattering by the quasi-particles in the medium. Furthermore, dissipative kernels of the sort treated here typically have some type of long time tail, which retains memory from the past. However, as will be discussed later, a non-vanishing decay width, as present in this case, helps to suppress the long time tail of the kernel.

Studies of terms similar to the second nonlocal term in Eq. (2.15) have been done in [26 28]. These works studied the case $N_{\psi}=1$ and $\Gamma_{\psi}=0$, and examined the linearized form of the effective EOM once the fermion fields were 
integrated out. An important issue raised in [27,28] was in regards the singularities in the EOM at the initial time, which in our case refers to the behavior of the dissipative kernel in Eq. (2.15) arising from the fermionic loop, when computed at $t=t^{\prime}$. Observe that this term appears divergent in the ultraviolet region, but this apparent problem easily can be solved in our approach. To see that our final equation in this case is identical to the final result obtained by the authors in [27,28], take for example the second nonlocal term in Eq. (2.15), due to the fermionic loop, and integrate the time integral twice by parts to give

$$
\begin{aligned}
& \sum_{k=1}^{N_{\psi}} h_{k}^{2} \int_{-\infty}^{t} d t^{\prime} \dot{\varphi}\left(t^{\prime}\right) \int \frac{d^{3} q}{(2 \pi)^{3}} \frac{e^{-2 \Gamma_{\psi_{k}}(q)\left|t-t^{\prime}\right|}}{\omega_{\psi_{k}}^{2} m_{\psi_{k}}^{2}\left(\Gamma_{\psi_{k}}^{2}+\omega_{\psi_{k}}^{2}\right)} \\
& \times\left\{\left[\Gamma_{\psi_{k}}^{4} \omega_{\psi_{k}}+2 \omega_{\psi_{k}}^{3} m_{\psi_{k}}^{2}-2 m_{\psi_{k}}^{4} \omega_{\psi_{k}}+\Gamma_{\psi_{k}}^{2} \omega_{\psi_{k}}\left(\omega_{\psi_{k}}^{2}+m_{\psi_{k}}^{2}\right)\right] \cos \left(2 \omega_{\psi_{k}}\left|t-t^{\prime}\right|\right)\right. \\
& \left.+\left[\Gamma_{\psi_{k}}\left(2 m_{\psi_{k}}^{2}-\Gamma_{\psi_{k}}^{2}\right)\left(\omega_{\psi_{k}}^{2}-m_{\psi_{k}}^{2}\right)-\Gamma_{\psi_{k}}^{5}\right] \sin \left(2 \omega_{\psi_{k}}\left|t-t^{\prime}\right|\right)\right\} \\
& =\sum_{k=1}^{N_{\psi}} h_{k}^{2} \dot{\varphi}(t) \int \frac{d^{3} q}{(2 \pi)^{3}} \frac{\left(\Gamma_{\psi_{k}}^{2}+2 \omega_{\psi_{k}}^{2}-2 m_{\psi_{k}}^{2}\right)}{\omega_{\psi_{k}}\left(\Gamma_{\psi_{k}}^{2}+\omega_{\psi_{k}}^{2}\right)^{2}} \Gamma_{\psi_{k}} \\
& +\sum_{k=1}^{N_{\psi}} h_{k}^{2} \ddot{\varphi}(t) \int \frac{d^{3} q}{(2 \pi)^{3}}\left[\frac{\omega_{\psi_{k}}^{2}-m_{\psi_{k}}^{2}}{2 \omega_{\psi_{k}}^{5}}+\mathcal{O}\left(\frac{\Gamma_{\psi_{k}}^{2}}{\omega_{\psi_{k}}^{2}}\right)\right] \\
& -\sum_{k=1}^{N_{\psi}} h_{k}^{2} \int_{-\infty}^{t} d t^{\prime} \dddot{\varphi}\left(t^{\prime}\right) \int \frac{d^{3} q}{(2 \pi)^{3}} e^{-2 \Gamma_{\psi_{k}}(q)\left|t-t^{\prime}\right|}\left\{\frac { \omega _ { \psi _ { k } } ^ { 2 } - m _ { \psi _ { k } } ^ { 2 } } { 2 \omega _ { \psi _ { k } } ^ { 5 } } \left[\cos \left(2 \omega_{\psi_{k}}\left|t-t^{\prime}\right|\right)\right.\right. \\
& \left.\left.+3 \frac{\Gamma_{\psi_{k}}}{\omega_{\psi_{k}}} \sin \left(2 \omega_{\psi_{k}}\left|t-t^{\prime}\right|\right)+\mathcal{O}\left(\frac{\Gamma_{\psi_{k}}^{2}}{\omega_{\psi_{k}}^{2}}\right)\right]\right\}
\end{aligned}
$$

The first term on the rhs of (2.20) is just the second dissipative term appearing in Eq. (2.18). By taking $\Gamma_{\psi_{k}}=0$ this term vanishes. The second term is a wave-function renormalization term, which can be absorbed in a wave function counterterm $(\delta Z)$ in the Lagrangian, by rewriting the kinetic term as $\left(\partial_{\mu} \Phi\right)^{2} \rightarrow(1+\delta Z)\left(\partial_{\mu} \Phi\right)^{2}$. The last term on the rhs of Eq. (2.20), for $\Gamma_{\psi_{k}}=0$ and $N_{\psi}=1$, reproduces exactly the result obtained in 27,28 for the EOM integrated over the fermion fields. Thus, we see that there is no ambiguities or divergences here associated with the kernels evaluated at equal times. Also, the non-local two-loop scalar term in Eq. (2.15) can be examined in a similar way and from such an analysis its contribution to the wave-function renormalization can be extracted.

Returning from this digression to the EOM Eq. (2.18), and using Eq. (2.19) for $\Gamma_{\phi}$ and $\Gamma_{\psi}=0$, the EOM becomes

$$
\ddot{\varphi}(t)+m_{\phi}^{2} \varphi(t)+\frac{\lambda}{6} \varphi^{3}(t)+\eta(\varphi) \dot{\varphi}(t)=0 .
$$

Here $\eta(\varphi)$ is the dissipative coefficients, which after doing the momentum integral in the "one-loop" dissipation term and using the symmetry of the "two-loop" dissipation term under change of momentum integration variables, becomes

$$
\begin{aligned}
\eta(\varphi) & =\varphi^{2}(t) \frac{\lambda^{2} \alpha_{\phi, \psi}^{2}}{128 \pi \sqrt{m_{\phi}^{4}+\alpha_{\phi, \psi}^{4}}\left(2 \sqrt{m_{\phi}^{4}+\alpha_{\phi, \psi}^{4}}+2 m_{\phi}^{2}\right)^{\frac{1}{2}}} \\
& +\frac{\lambda^{2} \alpha_{\phi, \psi}^{2}}{4} \int \frac{d^{3} \mathbf{q}_{1}}{(2 \pi)^{3}} \frac{d^{3} \mathbf{q}_{2}}{(2 \pi)^{3}}\left\{\frac{1}{\omega_{\phi}\left(\mathbf{q}_{1}\right)^{2} \omega_{\phi}\left(\mathbf{q}_{2}\right) \omega_{\phi}\left(\mathbf{q}_{1}+\mathbf{q}_{2}\right)\left[\omega_{\phi}\left(\mathbf{q}_{1}\right)+\omega_{\phi}\left(\mathbf{q}_{2}\right)+\omega_{\phi}\left(\mathbf{q}_{1}+\mathbf{q}_{2}\right)\right]^{3}}\right. \\
& \left.+\mathcal{O}\left(\frac{\Gamma_{\phi}^{2}}{\omega_{\phi}^{2}}\right)\right\}
\end{aligned}
$$

with

$$
\alpha_{\phi, \psi}^{2}=\sum_{k=1}^{N_{\psi}} \frac{h_{k}^{2}}{8 \pi} m_{\phi}^{2}\left(1-\frac{4 m_{\psi_{k}}^{2}}{m_{\phi}^{2}}\right)^{\frac{3}{2}}
$$

Finally, we also can easily work out an equivalent model of $\Phi$ coupled to bath fields that rather than fermionic fields are scalar fields, $\chi_{j}, j=1 \ldots N_{\chi}$, with a trilinear coupling as $\sum_{j=1}^{N_{\chi}} \frac{g_{j}^{2}}{2} \Phi \chi_{j}^{2}$. Once again, by choosing $m_{\phi}>2 m_{\chi_{j}}$ we 
have $\Gamma_{\chi_{j}}=0$ but a nonvanishing $\Phi$ decay width, $\Gamma_{\phi}$, which also has been evaluated in the Appendix. Going through the same steps as used to obtain Eq. (2.21), we obtain an analogous expression with a similar dissipative coefficient $\eta(\varphi)$, except with $\alpha_{\phi, \psi} \rightarrow \alpha_{\phi, \chi}$, where

$$
\alpha_{\phi, \chi}^{2}=\sum_{j=1}^{N_{\chi}} \frac{g^{4}}{16 \pi}\left(1-\frac{4 m_{\chi_{j}}^{2}}{m_{\phi}^{2}}\right)^{\frac{1}{2}}
$$

\section{B. Examination of the dissipative kernels}

Up to this point, the naive implementation of the Markovian approximation has been examined and has led from Eq. (2.15) to Eq. (2.21). The applicability of this approximation is now considered. Returning to the non-local EOM Eq. (2.15), we can express for example the term generating the one-loop dissipation contribution in Eq. (2.21) in the general formf

$$
\int_{-\infty}^{t} d t^{\prime} \varphi\left(t^{\prime}\right) \dot{\varphi}\left(t^{\prime}\right) K\left(t, t^{\prime}\right)
$$

where the dissipative kernel is given by

$$
K\left(t, t^{\prime}\right)=\lambda^{2} \int \frac{d^{3} q}{(2 \pi)^{3}} \frac{\left[\omega_{\phi} \cos \left(2 \omega_{\phi}\left|t-t^{\prime}\right|\right)+\Gamma_{\phi} \sin \left(2 \omega_{\phi}\left|t-t^{\prime}\right|\right)\right]}{8 \omega_{\phi}^{2}\left(\Gamma_{\phi}^{2}+\omega_{\phi}^{2}\right)} e^{-2 \Gamma_{\phi}(q)\left|t-t^{\prime}\right|} .
$$

In Fig. $1 K\left(t, t^{\prime}=0\right) / \lambda^{2}$ is plotted for four cases, $\Gamma_{\phi}(0) / m_{\phi}=0.1,0.5,1.0,5.0$. There is a degeneracy amongst the parameters $N_{\psi}, h$ and $m_{\psi}$ from which to choose for a given value of $\Gamma_{\phi}(0)$. The plots of the kernel are given over four different time interval, in order to see the different aspects of its behavior.

As can be seen, the kernel has a pronounced, narrow peak around $t=t^{\prime}$ followed by a power-law decaying oscillatory behavior, with greater decaying as the number of fields coupled to $\Phi$ increases. Since from Eq. (2.19) $\Gamma_{\phi} \sim 1 /|\mathbf{q}|$ for $|\mathbf{q}| \gg m_{\phi}$, it can be seen from Eq. (2.25) that once $|\mathbf{q}| \gtrsim 2 m_{\phi} \Gamma(0)\left|t-t^{\prime}\right|$, damping from the exponential term relinquishes. Thus for $\left|t-t^{\prime}\right| \gtrsim 1 / m_{\phi}$ the behavior of the kernel is $K\left(t, t^{\prime}\right) \sim-\lambda^{2} \operatorname{Ci}\left(4 m_{\phi} \Gamma_{\phi}(0)\left|t-t^{\prime}\right|^{2}\right) \sim$ $-\lambda^{2} \sin \left(4 m_{\phi} \Gamma_{\phi}(0)\left|t-t^{\prime}\right|^{2}\right) /\left(4 m_{\phi} \Gamma_{\phi}(0)\left|t-t^{\prime}\right|^{2}\right)$. Note this long time behavior of the $T=0$ contribution of the kernel differs from its high temperature component which was studied in [11,25]. At high temperature, the decay widths at large $|\mathbf{q}|$ behave as $\sim T^{2} /|\mathbf{q}|$, but there are also factors of the number density which become Boltzmann suppressed. As such, the high temperature limit of the kernel becomes highly exponentially suppressed at large times.

Power-law decay of the kernel at $T=0$ implies that memory of the scalar field is retained in Eq. (2.15) in determining its future evolution. As such, the derivative expansion clearly is not generally valid. However, for sufficiently slow motion of the scalar field, the derivative expansion still may be valid for some duration of time. In particular, suppose $\dot{\varphi} / \varphi \approx \gamma$ in a given solution, where $\gamma$ is the approximate magnitude of this ratio over some interval of time. Then, self-consistency of this solution with respect to the derivative expansion holds for a time interval $\left|t-t^{\prime}\right| \lesssim 1 / \gamma$. Thus, as mentioned at the beginning of this subsection, the slower is the motion of $\varphi$, the longer the above approximation is valid. Such slowly varying solutions are useful to investigate due to their simplicity. They also may have practical use for example for warm inflation, where one seeks solutions where the motion of $\varphi$ is slow.

Although the kernel Eq. (2.25) does retain past memory, it is worth noting that on general grounds for reasonable $\varphi(t)$ solution regimes, the memory retention only is up to some finite time in the past. The observation here is that the oscillation rate of the kernel increases with time due to the quadratic dependence on time $K\left(t, t^{\prime}\right) \sim \sin \left(a\left|t-t^{\prime}\right|^{2}\right)$, where $a$ is a constant. As such, for reasonable motions of $\varphi$, beyond some time interval into the past $\left|t-t^{\prime}\right|>\Delta t_{0}$, the characteristic oscillation frequency of the kernel will exceed that of $\varphi$. Thus at all times past this point, the contributions from $\varphi(t)$ primarily cancel. As a practical point, despite this property of the kernel to filter through increasingly high frequency components as the time passes, there are limitations to the types of $\varphi(t)$ motions which it can describe. In particular, this feature of the kernel most efficiently is able to cut-off the long time memory if

\footnotetext{
${ }^{4}$ Although here we analyze the one-loop dissipative kernel in detail, similar conclusions also can be shown to apply for the more complicate two-loop dissipative kernel in Eq. (2.15).
} 
the field configuration only has slow frequency components. As such, ideally the applicability of this kernel is near equilibrium conditions. Note, this also is the regime assumed in previous works [11, 12] for the finite temperature case.

An additional consistency condition related to the adiabatic approximation at finite $T$, considered in [12, 18], is that the microscopic dynamics should be much faster than all macroscopic motions. For the finite- $T$ case, this was a necessary requirement since it guaranteed the system thermalized fast enough to adjust to any changes in the macroscopic state. The need for rapid thermalization was necessary for self-consistency of the solutions. In contrast, the $T=0$ dynamics treated in this paper does not require a specific statistical state in which the scalar field evolution occurs. Thus, consistency requirements, if any, with respect to the rates of microscopic versus macroscopic dynamics are less well defined for the $T=0$ case. To properly address this question, a complete nonequilibrium analysis is necessary, which is beyond the scope of this paper. However, for the time being, to be conservative, a similar consistency condition to the finite- $T$ case adopted in [12] will be imposed, which requires the rate of microscopic physics to be faster than all macroscopic motions. Since the only scales characterizing the microscopic physics are the decay rates, this requirement implies

$$
\left|\frac{\varphi}{\dot{\varphi}}\right| \gg \Gamma^{-1}
$$

which is analogous to the condition in [12], except above the zero temperature decay rates are used. In Sec. IV some estimates will be given of dissipative dynamics based on the combined adiabatic-Markovian approximation of this and the previous subsections.

\section{INDIRECT DECAY MODELS}

This section will consider models in which the particles ultimately created from dissipation of the scalar field are coupled indirectly to the scalar field through an intermediate field. Such a case has much more variety in the types of decay sequences, as compared to the direct decay models of Sec. II. The basic model to be examined consists of the system, a scalar field $\Phi$, along with scalar fields $\chi_{j}, j=1 \ldots N_{\chi}$ and fermion fields $\psi_{k}, k=1 \ldots N_{\psi}$. The Lagrangian density is given by

$$
\begin{aligned}
\mathcal{L}\left[\Phi, \chi_{j}, \bar{\psi}_{k}, \psi_{k}\right] & =\frac{1}{2}\left(\partial_{\mu} \Phi\right)^{2}-\frac{m_{\phi}^{2}}{2} \Phi^{2}-\frac{\lambda}{4 !} \Phi^{4}+\sum_{j=1}^{N_{\chi}}\left\{\frac{1}{2}\left(\partial_{\mu} \chi_{j}\right)^{2}-\frac{m_{\chi_{j}}^{2}}{2} \chi_{j}^{2}-\frac{f_{j}}{4 !} \chi_{j}^{4}-\frac{g_{j}^{2}}{2} \Phi^{2} \chi_{j}^{2}\right\} \\
& +\sum_{k=1}^{N_{\psi}} \bar{\psi}_{k}\left[i \not \partial-m_{\psi_{k}}-h_{k, \phi} \Phi-\sum_{j=1}^{N_{\chi}} h_{k j, \chi} \chi_{j}\right] \psi_{k},
\end{aligned}
$$

where all coupling constants are positive: $\lambda, f_{j}, g_{j}^{2}, h_{k, \phi}, h_{k j, \chi}>0$.

As before, we are interested in obtaining the EOM for a scalar field configuration $\varphi=\langle\Phi\rangle$. For this, the fields $\chi_{j}$ and $\psi_{k}$ are regarded as part of the environment. Once again the scalar field $\Phi$ is decomposed into its expectation value and fluctuation, $\Phi=\varphi+\phi$, where $\langle\Phi\rangle=\varphi$. The EOM for $\varphi$ then is obtained from the tadpole method by imposing that $\langle\phi\rangle=0$, which leads to the condition that the sum of all tadpole terms vanish. Restricting again our analysis of the EOM to a homogeneous field $\varphi \equiv \varphi(t)$, we obtain the effective EOM for $\varphi$

$$
\begin{aligned}
& \ddot{\varphi}(t)+m_{\phi}^{2} \varphi(t)+\frac{\lambda}{6} \varphi^{3}(t)+\frac{\lambda}{2} \varphi(t)\left\langle\phi^{2}\right\rangle+\frac{\lambda}{6}\left\langle\phi^{3}\right\rangle+\sum_{j=1}^{N_{\chi}} g_{j}^{2}\left[\varphi(t)\left\langle\chi_{j}^{2}\right\rangle+\left\langle\phi \chi_{j}^{2}\right\rangle\right] \\
& +\sum_{k=1}^{N_{\psi}} h_{k, \phi}\left\langle\bar{\psi}_{k} \psi_{k}\right\rangle=0,
\end{aligned}
$$

where the field averages above can be expressed as usual in terms of the coincidence limit of the (causal) two-point Green's functions $G_{\phi}^{++}\left(x, x^{\prime}\right), G_{\chi}^{++}\left(x, x^{\prime}\right)$ and $S_{\psi}^{++}\left(x, x^{\prime}\right)$ for the $\Phi, \chi_{j}$ and $\psi_{k}$ fields, respectively. Using the expressions of the previous Section for $G^{++}$and $S^{++}$for the scalar and fermionic propagators respectively, and working out the expression analogous to Eq. (2.15), we obtain the EOM

$$
\ddot{\varphi}(t)+\bar{m}_{\phi}^{2} \varphi(t)+\frac{\bar{\lambda}}{6} \varphi^{3}(t)
$$




$$
\begin{aligned}
& +\lambda^{2} \varphi(t) \int_{-\infty}^{t} d t^{\prime} \varphi\left(t^{\prime}\right) \dot{\varphi}\left(t^{\prime}\right) \int \frac{d^{3} q}{(2 \pi)^{3}} \frac{\left[\omega_{\phi} \cos \left(2 \omega_{\phi}\left|t-t^{\prime}\right|\right)+\Gamma_{\phi} \sin \left(2 \omega_{\phi}\left|t-t^{\prime}\right|\right)\right]}{8 \omega_{\phi}^{2}\left(\Gamma_{\phi}^{2}+\omega_{\phi}^{2}\right)} e^{-2 \Gamma_{\phi}(q)\left|t-t^{\prime}\right|} \\
& +\frac{\lambda^{2}}{3} \int_{-\infty}^{t} d t^{\prime} \dot{\varphi}\left(t^{\prime}\right) \int \frac{d^{3} \mathbf{q}_{1}}{(2 \pi)^{3}} \frac{d^{3} \mathbf{q}_{2}}{(2 \pi)^{3}}\left\{\left[\omega_{\phi}\left(\mathbf{q}_{1}\right)+\omega_{\phi}\left(\mathbf{q}_{2}\right)+\omega_{\phi}\left(\mathbf{q}_{1}+\mathbf{q}_{2}\right)\right]\right. \\
& \times \cos \left[\left(\omega_{\phi}\left(\mathbf{q}_{1}\right)+\omega_{\phi}\left(\mathbf{q}_{2}\right)+\omega_{\phi}\left(\mathbf{q}_{1}+\mathbf{q}_{2}\right)\right)\left|t-t^{\prime}\right|\right] \\
& \left.+\left[\Gamma_{\phi}\left(\mathbf{q}_{1}\right)+\Gamma_{\phi}\left(\mathbf{q}_{2}\right)+\Gamma_{\phi}\left(\mathbf{q}_{1}+\mathbf{q}_{2}\right)\right] \sin \left[\left(\omega_{\phi}\left(\mathbf{q}_{1}\right)+\omega_{\phi}\left(\mathbf{q}_{2}\right)+\omega_{\phi}\left(\mathbf{q}_{1}+\mathbf{q}_{2}\right)\right)\left|t-t^{\prime}\right|\right]\right\} \\
& \times \frac{e^{-\left[\Gamma_{\phi}\left(\mathbf{q}_{1}\right)+\Gamma_{\phi}\left(\mathbf{q}_{2}\right)+\Gamma_{\phi}\left(\mathbf{q}_{1}+\mathbf{q}_{2}\right)\right]\left|t-t^{\prime}\right|}}{8 \omega_{\phi}\left(\mathbf{q}_{1}\right) \omega_{\phi}\left(\mathbf{q}_{2}\right) \omega_{\phi}\left(\mathbf{q}_{1}+\mathbf{q}_{2}\right)} \\
& \times \frac{1}{\left[\Gamma_{\phi}\left(\mathbf{q}_{1}\right)+\Gamma_{\phi}\left(\mathbf{q}_{2}\right)+\Gamma_{\phi}\left(\mathbf{q}_{1}+\mathbf{q}_{2}\right)\right]^{2}+\left[\omega_{\phi}\left(\mathbf{q}_{1}\right)+\omega_{\phi}\left(\mathbf{q}_{2}\right)+\omega_{\phi}\left(\mathbf{q}_{1}+\mathbf{q}_{2}\right)\right]^{2}} \\
& +\sum_{j=1}^{N_{\chi}} g_{j}^{4} \varphi(t) \int_{-\infty}^{t} d t^{\prime} \varphi\left(t^{\prime}\right) \dot{\varphi}\left(t^{\prime}\right) \int \frac{d^{3} q}{(2 \pi)^{3}} \frac{\left[\omega_{\chi_{j}} \cos \left(2 \omega_{\chi_{j}}\left|t-t^{\prime}\right|\right)+\Gamma_{\chi_{j}} \sin \left(2 \omega_{\chi_{j}}\left|t-t^{\prime}\right|\right)\right]}{2 \omega_{\chi_{j}}^{2}\left(\Gamma_{\chi_{j}}^{2}+\omega_{\chi_{j}}^{2}\right)} e^{-2 \Gamma_{\chi_{j}}\left|t-t^{\prime}\right|} \\
& +\sum_{j=1}^{N_{\chi}} g_{j}^{4} \int_{-\infty}^{t} d t^{\prime} \dot{\varphi}\left(t^{\prime}\right) \int \frac{d^{3} \mathbf{q}_{1}}{(2 \pi)^{3}} \frac{d^{3} \mathbf{q}_{2}}{(2 \pi)^{3}}\left\{\left[\omega_{\chi_{j}}\left(\mathbf{q}_{1}\right)+\omega_{\phi}\left(\mathbf{q}_{2}\right)+\omega_{\phi}\left(\mathbf{q}_{1}+\mathbf{q}_{2}\right)\right]\right. \\
& \times \cos \left[\left(\omega_{\chi_{j}}\left(\mathbf{q}_{1}\right)+\omega_{\phi}\left(\mathbf{q}_{2}\right)+\omega_{\phi}\left(\mathbf{q}_{1}+\mathbf{q}_{2}\right)\right)\left|t-t^{\prime}\right|\right] \\
& \left.+\left[\Gamma_{\chi_{j}}\left(\mathbf{q}_{1}\right)+\Gamma_{\phi}\left(\mathbf{q}_{2}\right)+\Gamma_{\phi}\left(\mathbf{q}_{1}+\mathbf{q}_{2}\right)\right] \sin \left[\left(\omega_{\chi_{j}}\left(\mathbf{q}_{1}\right)+\omega_{\phi}\left(\mathbf{q}_{2}\right)+\omega_{\phi}\left(\mathbf{q}_{1}+\mathbf{q}_{2}\right)\right)\left|t-t^{\prime}\right|\right]\right\} \\
& \times \frac{e^{-\left[\Gamma_{\chi_{j}}\left(\mathbf{q}_{1}\right)+\Gamma_{\phi}\left(\mathbf{q}_{2}\right)+\Gamma_{\phi}\left(\mathbf{q}_{1}+\mathbf{q}_{2}\right)\right]\left|t-t^{\prime}\right|}}{8 \omega_{\chi_{j}}\left(\mathbf{q}_{1}\right) \omega_{\phi}\left(\mathbf{q}_{2}\right) \omega_{\phi}\left(\mathbf{q}_{1}+\mathbf{q}_{2}\right)} \\
& \times \frac{1}{\left[\Gamma_{\chi_{j}}\left(\mathbf{q}_{1}\right)+\Gamma_{\phi}\left(\mathbf{q}_{2}\right)+\Gamma_{\phi}\left(\mathbf{q}_{1}+\mathbf{q}_{2}\right)\right]^{2}+\left[\omega_{\chi_{j}}\left(\mathbf{q}_{1}\right)+\omega_{\phi}\left(\mathbf{q}_{2}\right)+\omega_{\phi}\left(\mathbf{q}_{1}+\mathbf{q}_{2}\right)\right]^{2}} \\
& +\sum_{k=1}^{N_{\psi}} h_{k, \phi}^{2} \int_{-\infty}^{t} d t^{\prime} \dot{\varphi}\left(t^{\prime}\right) \int \frac{d^{3} q}{(2 \pi)^{3}} \frac{e^{-2 \Gamma_{\psi_{k}}(q)\left|t-t^{\prime}\right|}}{\omega_{\psi_{k}}^{2} m_{\psi_{k}}^{2}\left(\Gamma_{\psi_{k}}^{2}+\omega_{\psi_{k}}^{2}\right)} \\
& \times\left\{\left[\Gamma_{\psi_{k}}^{4} \omega_{\psi_{k}}+2 \omega_{\psi_{k}}^{3} m_{\psi_{k}}^{2}-2 m_{\psi_{k}}^{4} \omega_{\psi_{k}}+\Gamma_{\psi_{k}}^{2} \omega_{\psi_{k}}\left(\omega_{\psi_{k}}^{2}+m_{\psi_{k}}^{2}\right)\right] \cos \left(2 \omega_{\psi_{k}}\left|t-t^{\prime}\right|\right)\right. \\
& \left.+\left[\Gamma_{\psi_{k}}\left(2 m_{\psi_{k}}^{2}-\Gamma_{\psi_{k}}^{2}\right)\left(\omega_{\psi_{k}}^{2}-m_{\psi_{k}}^{2}\right)-\Gamma_{\psi_{k}}^{5}\right] \sin \left(2 \omega_{\psi_{k}}\left|t-t^{\prime}\right|\right)\right\}=0 .
\end{aligned}
$$

The dissipative dynamics in these indirect decay models will differ based on the relation of the masses amongst the $\Phi, \chi_{j}$ and $\psi_{i}$ fields. Consider first the case where the (effective) masses satisfy the relation

$$
m_{\phi}>m_{\chi_{j}}>2 m_{\psi_{k}}
$$

which we will refer to more specifically as the indirect cascade decay regime. The first attribute of this regime to note is at zero temperature $\Gamma_{\phi}$ and $\Gamma_{\chi_{j}}$ are non-vanishing and $\Gamma_{\psi}=0$. There are two kinematically allowed on-shell processes, $\Phi \rightarrow \psi_{k}+\bar{\psi}_{k}$, with decay width $\Gamma_{\phi}$ as given in Eq. (2.19), and $\chi_{j} \rightarrow \psi_{k}+\bar{\psi}_{k}$, with decay width

$$
\Gamma_{\chi_{j}}(q)=\sum_{k=1}^{N_{\psi}} \frac{h_{k j, \chi}^{2}}{8 \pi \omega_{\chi_{j}}} m_{\chi_{j}}^{2}\left(1-\frac{4 m_{\psi_{k}}^{2}}{m_{\chi_{j}}^{2}}\right)^{\frac{3}{2}} .
$$

Implementing once again the adiabatic-Markovian approximation of Subsec. IIA, we then obtain the same expression for the EOM as Eq. (2.21), except $\eta(\varphi)$ now is given by

$$
\begin{aligned}
& \eta(\varphi)=\varphi^{2}(t) \frac{\lambda^{2} \alpha_{\phi, \psi}^{2}}{128 \pi \sqrt{m_{\phi}^{4}+\alpha_{\phi, \psi}^{4}} \sqrt{2 \sqrt{m_{\phi}^{4}+\alpha_{\phi, \psi}^{4}}+2 m_{\phi}^{2}}} \\
& +\frac{\lambda^{2} \alpha_{\phi, \psi}^{2}}{4} \int \frac{d^{3} \mathbf{q}_{1}}{(2 \pi)^{3}} \frac{d^{3} \mathbf{q}_{2}}{(2 \pi)^{3}}\left\{\frac{1}{\omega_{\phi}\left(\mathbf{q}_{1}\right)^{2} \omega_{\phi}\left(\mathbf{q}_{2}\right) \omega_{\phi}\left(\mathbf{q}_{1}+\mathbf{q}_{2}\right)\left[\omega_{\phi}\left(\mathbf{q}_{1}\right)+\omega_{\phi}\left(\mathbf{q}_{2}\right)+\omega_{\phi}\left(\mathbf{q}_{1}+\mathbf{q}_{2}\right)\right]^{3}}+\mathcal{O}\left(\frac{\Gamma_{\phi}^{2}}{\omega_{\phi}^{2}}\right)\right\}
\end{aligned}
$$




$$
\begin{aligned}
& +\varphi^{2}(t) \sum_{j=1}^{N_{\chi}} g_{j}^{4} \frac{\alpha_{\chi, \psi}^{2}}{32 \pi} \frac{1}{\sqrt{m_{\chi_{j}}^{4}+\alpha_{\chi, \psi}^{4}} \sqrt{2 \sqrt{m_{\chi_{j}}^{4}+\alpha_{\chi, \psi}^{4}}+2 m_{\chi_{j}}^{2}}} \\
& +\sum_{j=1}^{N_{\chi}} \frac{g_{j}^{4} \alpha_{\chi, \psi}^{2}}{4} \int \frac{d^{3} \mathbf{q}_{1}}{(2 \pi)^{3}} \frac{d^{3} \mathbf{q}_{2}}{(2 \pi)^{3}}\left\{\frac{1}{\omega_{\chi_{j}}\left(\mathbf{q}_{1}\right)^{2} \omega_{\phi}\left(\mathbf{q}_{2}\right) \omega_{\phi}\left(\mathbf{q}_{1}+\mathbf{q}_{2}\right)\left[\omega_{\chi_{j}}\left(\mathbf{q}_{1}\right)+\omega_{\phi}\left(\mathbf{q}_{2}\right)+\omega_{\phi}\left(\mathbf{q}_{1}+\mathbf{q}_{2}\right)\right]^{3}}\right. \\
& \left.+\mathcal{O}\left(\frac{\Gamma_{\chi_{j}}^{2}, \Gamma_{\phi}^{2}}{\omega^{2}}\right)\right\},
\end{aligned}
$$

with $\alpha_{\phi, \psi}$ given by Eq. 2.23) and

$$
\alpha_{\chi, \psi}^{2}=\sum_{k=1}^{N_{\psi}} \frac{h_{k j, \chi}^{2}}{8 \pi} m_{\chi_{j}}^{2}\left(1-\frac{4 m_{\psi_{k}}^{2}}{m_{\chi_{j}}^{2}}\right)^{\frac{3}{2}} .
$$

It is interesting to note that the last two terms in Eq. $(3.6)$ come solely from the decay channels of $\chi_{j}$ into the fermion fields $\psi_{k}$, which then backreact on the system field $\varphi$ in terms of a damping force. The same effect also would appear for the case where $\Phi$ was the lightest field,

$$
m_{\chi_{j}}>2 m_{\psi_{k}}>m_{\phi}
$$

This regime will be referred to simply as the indirect decay regime outside the cascade region Eq. (3.4). For this case $\Gamma_{\phi}=0$, or $\alpha_{\phi, \psi}=0$ in Eq. (3.6). This type of dissipation, in which the system field is lighter than the decay products was first noted by Calzetta and $\mathrm{Hu}$ in [14]. They have shown how a heavy field influences the dynamics of a light field in the form of dissipation and fluctuations of the light field, even when no aspect of the light field dynamics is above the mass threshold of the heavy field. This same behavior also can be inferred from our results for the above regime.

Let us finally make a few comments about higher order loop terms. Observe that the results obtained for the dissipation coefficients in Eqs. (2.22) and (3.6) are the leading order ones at zero temperature, $\mathcal{O}\left(\lambda^{2} h_{k}^{2}\right)$ and $\mathcal{O}\left(g_{j}^{4} h_{k j, \chi}^{2}\right)$ respectively. We have neglected higher order loop contributions to the EOM, since they all can be shown also to be of higher order in the coupling constants. For instance, a two-loop contribution made of a fermion loop with a vertical scalar propagator can easily be seen to give a contribution to the dissipation coefficient $\eta(\varphi)$ of order $\mathcal{O}\left(h_{k}^{4} \Gamma_{\phi}\right) \sim \mathcal{O}\left(h_{k}^{6}\right)$, for the first case analyzed in Sec. I], and $\mathcal{O}\left(h_{k, \phi}^{2} h_{k j, \chi}^{4}\right)$, for the case studied in this section. Also, higher order scalar loop terms are subleading in the coupling constants, as compared to the results given by Eqs. (2.22) and (3.6). For example, at finite temperature, scalar ladder diagrams [12,29] are known to be of the same order as the twovertex one-loop term in the EOM, due to on-shell divergences of these diagrams and the way the field decay widths regularize them. However at zero temperature these divergences are not present and ladder diagrams are at most of order $\mathcal{O}\left(\lambda^{4} \Gamma_{\phi}^{2}\right) \sim \mathcal{O}\left(\lambda^{4} h_{k}^{4}\right)$, for the model in Sec. II and $\mathcal{O}\left(g_{j}^{8} h_{k j, \chi}^{4}\right)$, for the model in this section.

\section{APPLICATION}

In this Section we will apply the effective equation of motion Eq. (2.21), with $\eta(\varphi)$ as given by Eqs. (2.22) and (3.6), to make estimates of entropy production from conversion of the scalar field potential energy into radiation. Recall the applicability of Eq. (2.21) is limited, since it requires the validity of the adiabatic-Markovian approximation of Sect. II. For this reason and since as mentioned in the Introduction, the zero temperature dissipation found here is indicative of a nonequilibrium dynamics that drives the system to finite temperature, we will not delve into detailed applications of this effective equation of motion. The full dynamics of this problem must be understood before detailed application is worthwhile. Nevertheless, we will make some naive estimates of radiation production from our equations, just to get a feeling for the magnitudes of the effect. Note that for application to warm inflation, the fact dissipation occurs at $T=0$ implies the dynamics automatically will bootstrap the universe to finite temperature, independent of initial conditions.

In general for a damped equation of motion of the form (2.21) the regime of overdamped (underdamped) motion is

$$
m^{2}(\varphi)=m_{\phi}^{2}+\frac{\lambda}{2} \varphi^{2}<(>) \eta^{2}(\varphi)
$$

For the direct decay models in Sec. II, since the dissipative coefficient $\eta(\varphi)$ is suppressed by two powers of the $\phi$ selfcoupling parameter $\lambda$, we find that underdamped motion generally is possible, unless there are very many environment 
bath fields. For the indirect decay models of Sect. III, the cascade decay regime Eq. (3.4) has similar constraints, thus solutions, as those of the direct decay models of Sect. [1. However, in the other interesting regime Eq. (3.8), the dissipative coefficient and the parameters of the $\varphi$ potential can be independently tuned, which means the overdamped regime can be identified for as few as one or two heat bath fields. Both the underdamped and overdamped regimes may have application to the chiral transition in heavy ion collision [30], whereas the overdamped regime also is of interest to the warm inflation scenario [21]. Below, both the direct and indirect decay models will be examined.

For the direct decay model, the overdamped (underdamped) regime from Eqs. (2.22), (2.23) and (4.1) is given by

$$
\frac{\lambda^{2} N_{\psi}}{2048 \pi^{2}} \gtrsim(\lesssim) 1,
$$

where the Yukawa coupling $h$ is determined by requiring $m_{\psi} \approx h \varphi<m(\varphi) / 2 \approx \sqrt{\lambda} \varphi / 2$. Thus, even for strong coupling $\lambda \sim 1$, it requires $N_{\psi} \gtrsim 10^{4}$ fields for the overdamped regime.

Considering first the underdamped regime, the adiabatic condition Eq. (2.26) requires

$$
\frac{\dot{\varphi}}{\varphi} \approx m(\varphi)<\Gamma_{\psi} \approx \frac{h^{2} N_{\psi}}{8 \pi} m(\varphi) \approx \frac{\lambda N_{\psi}}{16 \pi} m(\varphi),
$$

where for underdamped motion from Eq. (2.21) $\dot{\varphi} \lesssim m(\varphi) \varphi$. The energy dissipated by the scalar field goes into radiation energy density $\rho_{r}$, here composed of fermions and/or scalar bosons, at the rate

$$
\dot{\rho}_{r}=-\frac{d E_{\phi}}{d t}=\eta(\varphi) \dot{\varphi}^{2} \approx \frac{\lambda^{2} N_{\psi} m^{5}(\varphi)}{2048 \pi^{2}},
$$

where to obtain the last expression we estimate $\dot{\varphi}^{2} \approx m^{2}(\varphi) \varphi^{2}$. For the overdamped regime from Eq. (2.21) $\dot{\varphi}=m^{2}(\varphi) \varphi / \eta(\varphi)$. Writing $\eta(\varphi)=Q m(\varphi)$, from Eqs. (2.22) and (2.23) $Q \approx \lambda^{2} N /\left(2048 \pi^{2}\right)$ and for overdamping Eq. (4.1) requires $Q>1$. This requirement of overdamping automatically implies the adiabatic condition Eq. (2.26) is satisfied. Thus the radiation production in this case is

$$
\dot{\rho}_{r} \approx \frac{m^{3}(\varphi) \varphi^{2}}{Q} .
$$

Overall, for the direct decay model, the underdamped regime is generic except if there are a very large number of bath fields $N>10^{4}$, in which case overdamped motion also becomes possible. In the underdamped regime, moderate radiation energy production occurs. In particular during a characteristic oscillation time $\sim 1 / m(\varphi)$, the produced radiation has an associated temperature scale $T \approx\left(\rho_{r} / N_{\psi}\right)^{1 / 4} \approx \lambda^{1 / 2} m(\varphi) / 10 \lesssim m(\varphi)$. On the other hand, the overdamped regime, although requiring a large number of bath fields, can yield sizable radiation by increasing the amplitude $\varphi$ in Eq. (4.5)

Turning next to the indirect decay models of Sec. [II], the cascade region Eq. (3.4) leads to similar solutions as given above for the direct decay models, so will not be further elaborated. However, dissipative dynamics also appears to occur in the regime Eq. (3.8), which does not have a direct interpretation in terms of particle decay at one of the two steps of the process, the $\varphi \rightarrow \chi$ transition. We believe further investigation is needed of this case in order to obtain a sensible interpretation of this process, and this is left for future work. Nevertheless, here it is interesting to estimate the size of the dissipative effects for this case. In particular for this case, the overdamped regime can easily be obtained as will be shown next. The overdamped regime requires the condition in Eq. (1.1) and the adiabatic condition requires

$$
\frac{\dot{\varphi}}{\varphi}=\frac{m^{2}(\varphi)}{\eta(\varphi)}<\Gamma_{\chi}
$$

Since the scalar field sector has two free parameters, $m_{\phi}$ and $\lambda$, it is always possible to tune $m^{2}(\varphi)$ to satisfy both the above requirements independent of $\Gamma_{\chi}, \eta(\varphi)$, and for any amplitude $\varphi$.

In the overdamped regime, the kinetic energy of the scalar field is negligible. Thus, the loss in its potential energy translates into the energy released into radiation $\rho_{r}$ as

$$
\begin{aligned}
\dot{\rho}_{r}(t) & =\eta(\varphi) \dot{\phi}^{2}=-\frac{d V}{d \varphi} \dot{\varphi} \\
& =\left(m_{\phi}^{2} \varphi+\frac{\lambda}{6} \varphi^{3}\right) \dot{\varphi} \approx V(\varphi) \frac{m^{2}(\varphi)}{\eta}<V(\varphi) \Gamma_{\chi},
\end{aligned}
$$


where the second line follows from Eqs. (2.21) and (3.1). Generally the microscopic scale is determined by $\Gamma_{\chi}$. In this time interval, we find the radiation to increase to

$$
\rho_{r}\left(1 / \Gamma_{\chi}\right) \approx V(\varphi) \frac{m^{2}(\varphi)}{\eta \Gamma_{\chi}}<V(\varphi)
$$

where to obtain the right most expression we used Eq. (4.6). Thus the energy dissipated into radiation is proportional to the potential energy contained in the scalar field. To consider some numbers, for example typical for inflation, suppose the potential energy is at the GUT scale $V(\varphi)^{1 / 4} \sim 10^{15-16} \mathrm{GeV}$ and $m^{2}(\varphi) /\left(\eta \Gamma_{\chi}\right) \approx 10^{-4}$. For this, it implies a radiation component is generated which, if expressed in terms of temperature, is at the scale $T \sim 10^{14-15} \mathrm{GeV}$, and this is nonnegligible. The main point to note is that considerable radiation production can occur from the indirect decay models in the regime Eq. (3.8).

\section{CONCLUSION}

Although the calculations in this paper were for non-expanding Minkowski spacetime, a brief reflection will be made here on the consequences of these results for inflationary cosmology. In this case, the background space is expanding. The effect of the interaction between the scalar field, in this case called the inflaton, and the background metric is known to yield a $3 H \dot{\varphi}$ term in the inflaton effective equation of motion, where the Hubble parameter $H \equiv \dot{a} / a$ and $a(t)$ is the cosmic scale factor. As well known, this term does not arise from microscopic interactions with other fields, but rather from the macroscopic interaction with the background metric of gravity. Precisely this disparity in scales and the difference in origin of the interactions suggests that the effect of this term and the dissipative term computed in this paper will act independently on the inflaton to a good approximation, with perhaps some self-consistency requirements. At present, we are extending our calculation to expanding spacetime in order to examine this point. Should this work render true the expectations from Sec. IV for dissipation, it will be difficult to justify the supercooled inflation picture and rather it would appear the warm inflation picture is the natural one.

For the moment, assuming the correctness of the above expectations, a lower bound on the temperature of the universe during inflation can be estimated from the direct decay model. In typical inflation models, $m_{\phi} \sim H$ for supercooled inflation and $m_{\phi}$ perhaps a few orders of magnitude bigger than $H$ for warm inflation. Furthermore, the conditions on density perturbations generally require the inflaton self-coupling parameter to be tiny $\lambda \sim 10^{-(10-16)}$. In an expanding background, the evolution of the radiation in presence of a dissipating scalar field source is given by Eq. (4.3) with the addition of the term $-4 H \rho_{r}$ to the right hand side, which accounts for the redshift of the radiation due to background expansion. Therefore, in steady state $\dot{\rho}_{r}=0, \rho_{r} \approx \eta \dot{\varphi}^{2} /(4 H)$, or the associated temperature $T \sim\left(\rho_{r} / g^{*}\right)^{1 / 4}$, where we will take the number of light particles $g^{*} \sim N$. With these estimates, based on Eq. (4.3) we find $T \gtrsim \lambda^{1 / 2} m_{\phi} / 10$. If $m_{\phi} \sim 10^{10} \mathrm{GeV}$ as a typical value, then this implies $T \sim\left(10^{4}-10\right) \mathrm{GeV}$.

In order not to affect the successful predictions of nucleosynthesis, the primary requirement is that the universe should be well within the hot Big-Bang radiation dominated regime by $T \approx 10 \mathrm{MeV}$. Slightly more conservative, though not necessarily mandatory, is to require that the QCD phase transition at $T \lesssim 1 \mathrm{GeV}$ occurs within the radiation dominated era. So a safe lower bound for inflation to end and the radiation dominated era to commence is $T \gtrsim 1 \mathrm{GeV}$. As such, the lower limits for radiation production during inflation given above still would be above this lower limit requirement set by cosmology. The results found here may be also useful in applications to the low temperature regimes of warm inflation identified in the phenomenological studies of [31].

It should be clarified that the results found in this paper in no way require supersymmetry. However, these calculations easily could be applied in SUSY models. In such models, it is becoming appreciated that to avoid gravitino overproduction, for any type of inflation scenario the temperature of the universe after inflation can not be very high $T \lesssim 10^{10} \mathrm{GeV}[32]$. From this perspective, the possibility found in this paper for low temperature warm inflation solutions in the direct decay models would be phenomenologically attractive.

One cautionary remark is in order. Since $m_{\phi} \sim H$, the lower limit on the temperature during inflation that is suggested above, is below the so called Gibbons-Hawking "temperature" $T_{G H}=H /(2 \pi)$. However $T_{G H}$ does not represent a temperature in the usual sense of a thermal bath of particles. $T_{G H}$ acts like a temperature in the formal sense that for a non-interacting scalar field in de Sitter space, its euclideanized de Sitter invariant Green's function is periodic in imaginary time. The role that $T_{G H}$ plays in this Green's function is formally the same as what actual temperature plays in the static thermal Green's function in Minkowski space. However, for the non-interacting scalar 
field in the de Sitter case, there are no particles present in the sense that the field is in a vacuum state. In contrast, the radiation production we have computed for the interacting scalar field results in real particle production, irrespective whether its associated temperature is above or below $T_{G H}$.

In summary, this paper has studied dissipative effects of interacting scalar fields at zero temperature. Similar treatments along this line are limited [2, 13, 14] and one of the novel features of this paper is the appropriate inclusion of quasi-particles effects through the fully dressed zero temperature two-point Green's functions. Another feature of our analysis which has been studied only to a limited extent in the literature is a detailed examination of the dissipative kernel in Subsec. IIB. The models examined in this paper were generic and in all cases dissipation was found. Since dissipative effects are seen for the zero temperature state, we conclude that radiation production from dissipation is invariably present for generic interacting scalar field systems, although the extent of radiation production can vary immensely. Minimally, it appears the mass of the scalar field times a suitable dimensionless coupling constant sets a lower limit to the associated temperature scale of the produced radiation. However, for the indirect decay models in the region Eq. (3.8), there appears a much more robust possibility for producing radiation. Although formally this is what is indicated by our calculations, as mentioned earlier, we feel further investigation is necessary of these indirect decay models in order to obtain a sensible interpretation of its dissipative process. In regards the potential implications of the results found in this paper to inflationary cosmology, we infer that under generic circumstances the scalar inflaton field will dissipate a nonnegligible amount of radiation during inflation. In particular, the lower bound suggested by the above estimates for the direct decay models already are sufficiently high to preclude a mandatory requirement for a reheating. Furthermore, the upper bound from the indirect decay models in the regime Eq. (3.8) could yield very high temperature warm inflation solutions, in the range discussed below Eq. (4.8). However, these only are expectations suggested by the calculations in this paper. Verification of these expectations requires a proper extension of these calculations to expanding spacetime, which we currently are examining.

\section{ACKNOWLEDGMENTS}

We thank Larry Ford for helpful discussions. We especially thank Ian Lawrie for several discussions and for a careful examination of our paper. AB was funded by the United Kingdom Particle Physics and Astronomy Research Council (PPARC) and ROR was supported by Conselho Nacional de Desenvolvimento Científico e Tecnológico (CNPq-Brazil) and SR2-UERJ.

\section{APPENDIX A:}

In this appendix the decay widths for the processes $\phi \rightarrow \psi+\bar{\psi}$ (or $\chi \rightarrow \psi+\bar{\psi}$ ) and $\phi \rightarrow \chi+\chi$ are derived. Recall the basic expression for the decay of an initial particle of momentum $p$ into two particles,

$$
\begin{aligned}
\Gamma(p)= & \frac{1}{2 \omega_{\mathbf{p}}}\left[\int \frac{d^{4} k_{1}}{(2 \pi)^{3}} \frac{d^{4} k_{2}}{(2 \pi)^{3}}(2 \pi)^{4} \delta^{(4)}\left(k_{1}+k_{2}-p\right)\right. \\
& \left.\times \delta\left(k_{1}^{2}-m_{1}^{2}\right) \delta\left(k_{2}^{2}-m_{2}\right) \prod_{\text {fermion }_{j}}\left(2 m_{j}\right) \sum_{\operatorname{spins}_{j}}\left|\mathcal{M}_{f i}\right|^{2}\right],
\end{aligned}
$$

where $\omega_{\mathbf{p}}=\sqrt{\mathbf{p}^{2}+M^{2}}, M$ is the mass of the scalar decaying field, and $m$ stands for the mass of the decay products. Observe that the expression in the square brackets is Lorentz invariant, thus most conveniently it is evaluated in the rest frame of the initial particle $\mathbf{p}=0$.

For the scalar to 2 fermion model ( $h$ is the Yukawa coupling)

$$
\mathcal{L}_{I}=-h \phi(x) \bar{\psi}(x) \psi(x),
$$

which implies

$$
\mathcal{M}=-i h \bar{u}_{s_{1}}\left(\mathbf{k}_{1}\right) v_{s_{2}}\left(\mathbf{k}_{2}\right),
$$

${ }^{5}$ We thank Larry Ford for this clarification 
so that

$$
\sum_{s_{j}}|\mathcal{M}|^{2}=h^{2} \frac{k_{1} \cdot k_{2}-m^{2}}{m^{2}} .
$$

Substituting this into Eq. (A1) gives

$$
\Gamma(p)=\frac{h^{2} M^{2}}{8 \pi \omega(\mathbf{p})}\left(1-\frac{4 m^{2}}{M^{2}}\right)^{3 / 2}
$$

For the scalar to two scalar model, with coupling constant $g$, we have instead that

$$
\mathcal{L}_{I}=-\frac{g^{2}}{2} \phi(x) \chi^{2}(x)
$$

which implies

$$
\mathcal{M}=-i g^{2}
$$

and so

$$
\Gamma(p)=\frac{g^{4}}{16 \pi \omega_{\phi}(\mathbf{p})}\left(1-\frac{4 m^{2}}{M^{2}}\right)^{1 / 2}
$$

[1] D. Boyanovsky and H. J. de Vega, hep-ph/9909372.

[2] D. Boyanovsky, H. J. de Vega, R. Holman, D. S-Lee and A. Singh, Phys. Rev. D51, 4419 (1995).

[3] D. Boyanovsky, M. D'attanasio, H.J. de Vega, R. Holman and D.-S.Lee, Phys. Rev. D52, 6805 (1995).

[4] D. Boyanovsky, H. J. de Vega, R. Holman and J. F. J. Salgado, Phys. Rev. D54, 7570 (1996); Phys. Rev. D59, 125009 (1999).

[5] F. Cooper, S. Habib, Y. Kluger and E. Mottola, Phys. Rev. D55, 6471 (1997).

[6] S. A. Ramsey and B. L. Hu, Phys. Rev. D56, 661 (1997).

[7] J. Baacke, K. Heitmann and C. Patzold, Phys. Rev. D57, 6406 (1998).

[8] A. Hosoya and M. Sakagami, Phys. Rev. D29, 2228 (1984).

[9] M. Morikawa, Phys. Rev. D33, 3607 (1986). M. Morikawa and M. Sasaki, Phys. Lett. 165B, 59 (1985).

[10] I. D. Lawrie, J. Phys. A25, 6493 (1992); Phys. Rev. D60, 063510 (1999);

[11] M. Gleiser and R. O. Ramos, Phys. Rev. D50, 2441 (1994).

[12] A. Berera, M. Gleiser and R. O. Ramos, Phys. Rev. D58, 123508 (1998).

[13] E. Calzetta and B. L. Hu, Phys. Rev. D61, 025012 (2000); Phys. Rev. D40, 656 (1989).

[14] E. Calzetta and B. L. Hu, Phys. Rev. D55, 3536 (1997).

[15] Sz. Borsanyi, A. Patkos, J. Polonyi and Zs. Szep, Phys. Rev. D62, 085013 (2000).

[16] G. Aarts, G. F. Bonini and C. Wetterich, Nucl. Phys. B587, 403 (2000).

[17] A. O. Caldeira and A. J. Leggett, Ann. Phys. 149, 374 (1983).

[18] A. Berera, M. Gleiser and R. O. Ramos, Phys. Rev. Lett. 83, 264 (1999).

[19] A. Berera, Nucl. Phys. B585, 666 (2000).

[20] J. Yokoyama and A. Linde, Phys. Rev. D60, 083509 (1999).

[21] A. Berera, Phys. Rev. Lett. 75, 3218 (1995); Phys. Rev. D54, 2519 (1996); Phys. Rev. D55, 3346 (1997).

[22] G. Semenoff and N. Weiss, Phys. Rev. D31, 699 (1985); A. Ringwald, Phys. Rev. D36, 2598 (1987); Ann. Phys. 177, 129 (1987); Z. Phys. C34, 481 (1987).

[23] I. D. Lawrie and D. B. McKernan Phys. Rev. D62, 105032 (2000).

[24] C. Greiner and B. Müller, Phys. Rev. D55, 1026 (1997).

[25] R. O. Ramos and F. A. R. Navarro, Phys. Rev. D62, 085016 (2000).

[26] S. A. Ramsey, B. L. Hu and A. M. Stylianopoulos, Phys. Rev. D57, 6003 (1998).

[27] J. Baacke, K. Heitmann and C. Patzold, Phys. Rev. D58, 125013 (1998).

[28] J. Baacke, D. Boyanovsky and H. J. de Vega, hep-ph/9907337. 
[29] S. Jeon, Phys. Rev. D47, 4586 (1993); Phys. Rev. D52, 3591 (1995).

[30] for a review of the chiral phase transition please see K. Rajagopal, in Quark-Gluon Plasma II, ed. R. Hwa, World Scientific (1995), hep-ph/9504310; see also Z. Xu and C. Greiner, Phys Rev. D62, 036012 (2000).

[31] H. P. de Oliveira and R. O. Ramos, Phys. Rev. D57, 741 (1998); W. Lee and L.-Z. Fang, Phys. Rev. D59, 083503 (1999).

[32] M. Kawasaki and T. Moroi, Prog. Theor. Phys. 93, 879 (1995). 


\section{Figure Caption}

Fig. 1. The kernel $K(t, 0) / \lambda^{2}$ in Eq. (2.25) for the cases $\Gamma_{\phi}(0) / m_{\phi}=0.1$ (solid), 0.5 (dashed), 1.0 (dotted), and 5.0 (dot-dashed), plotted over four different time intervals. 


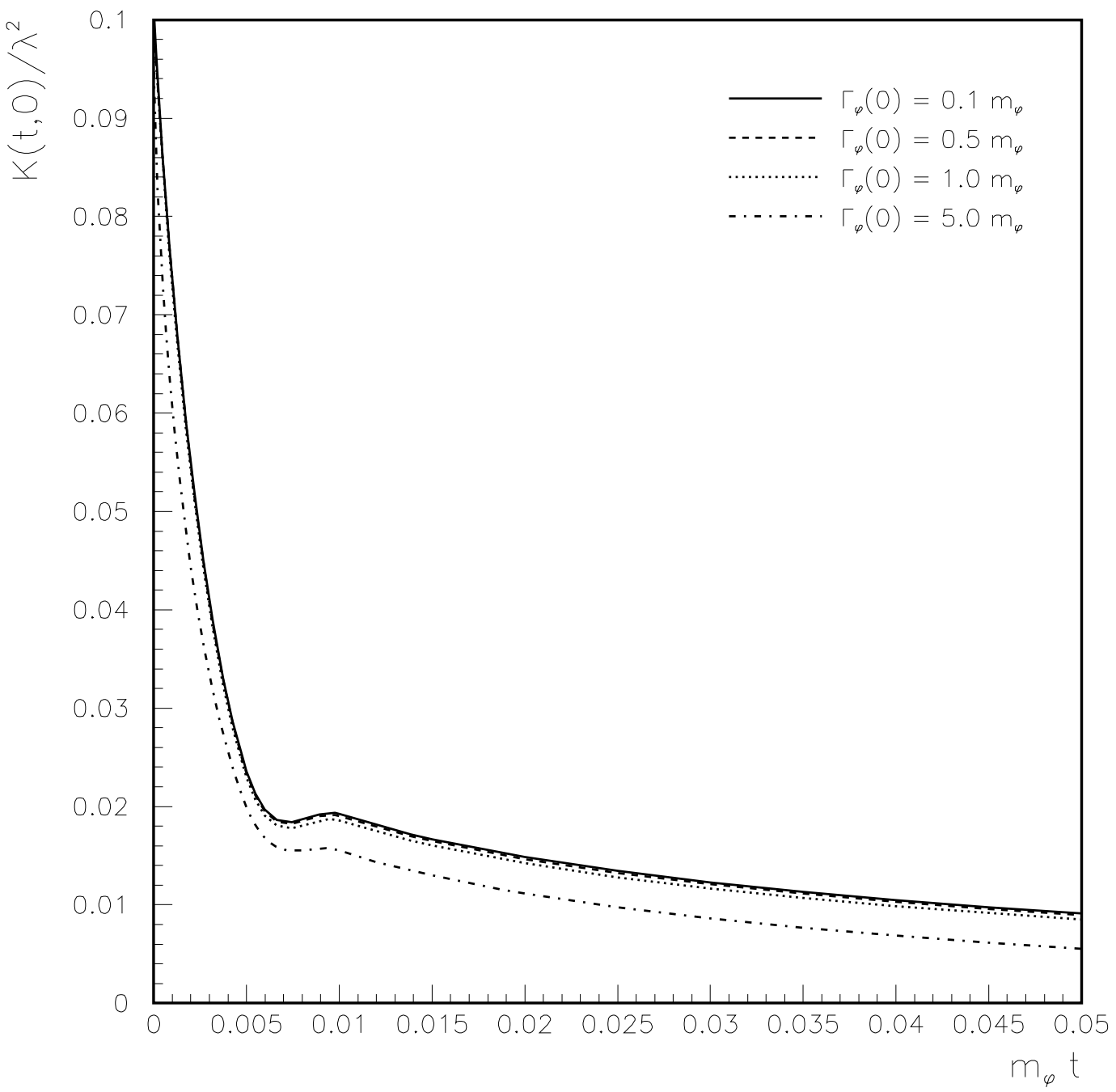




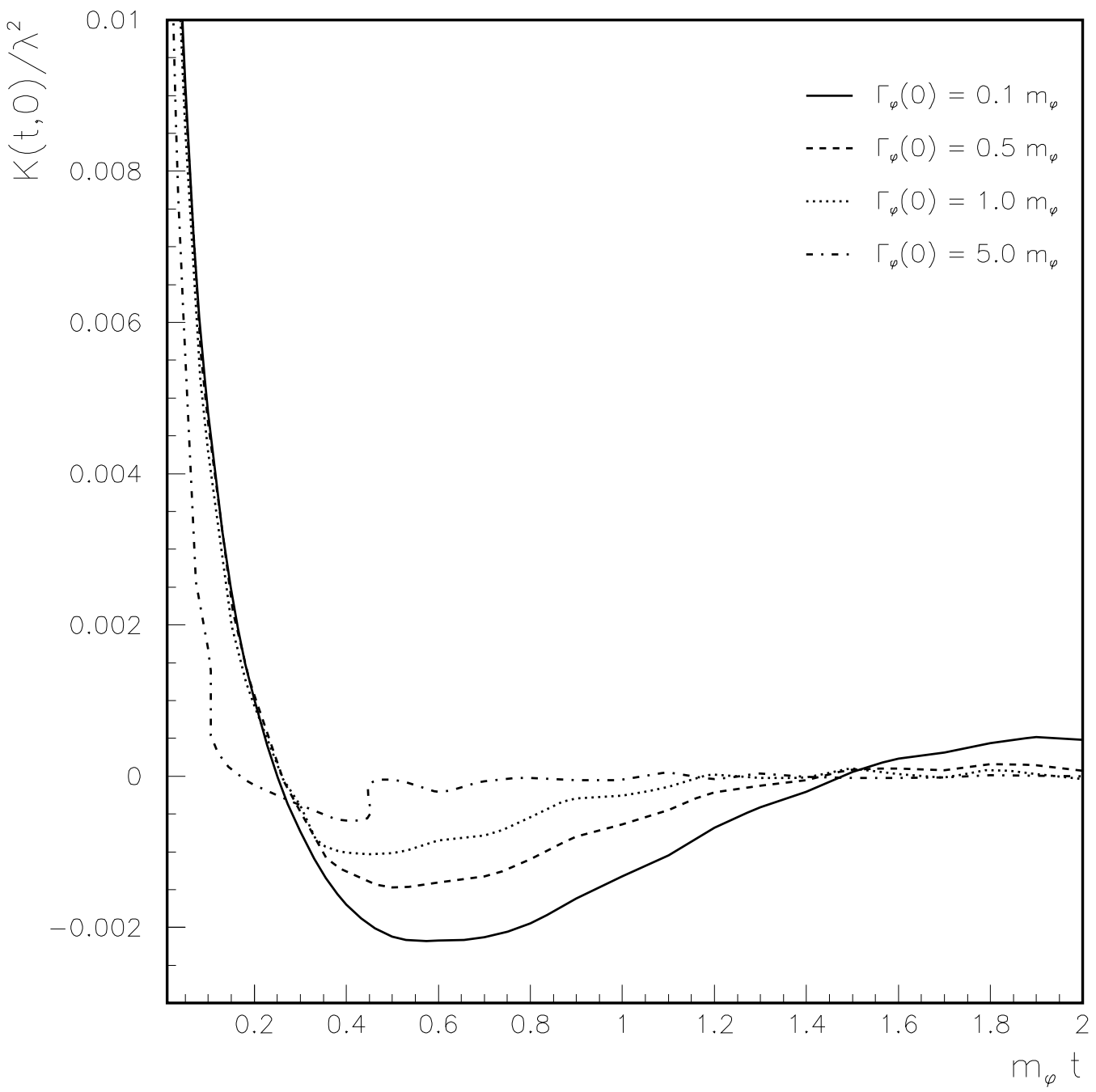




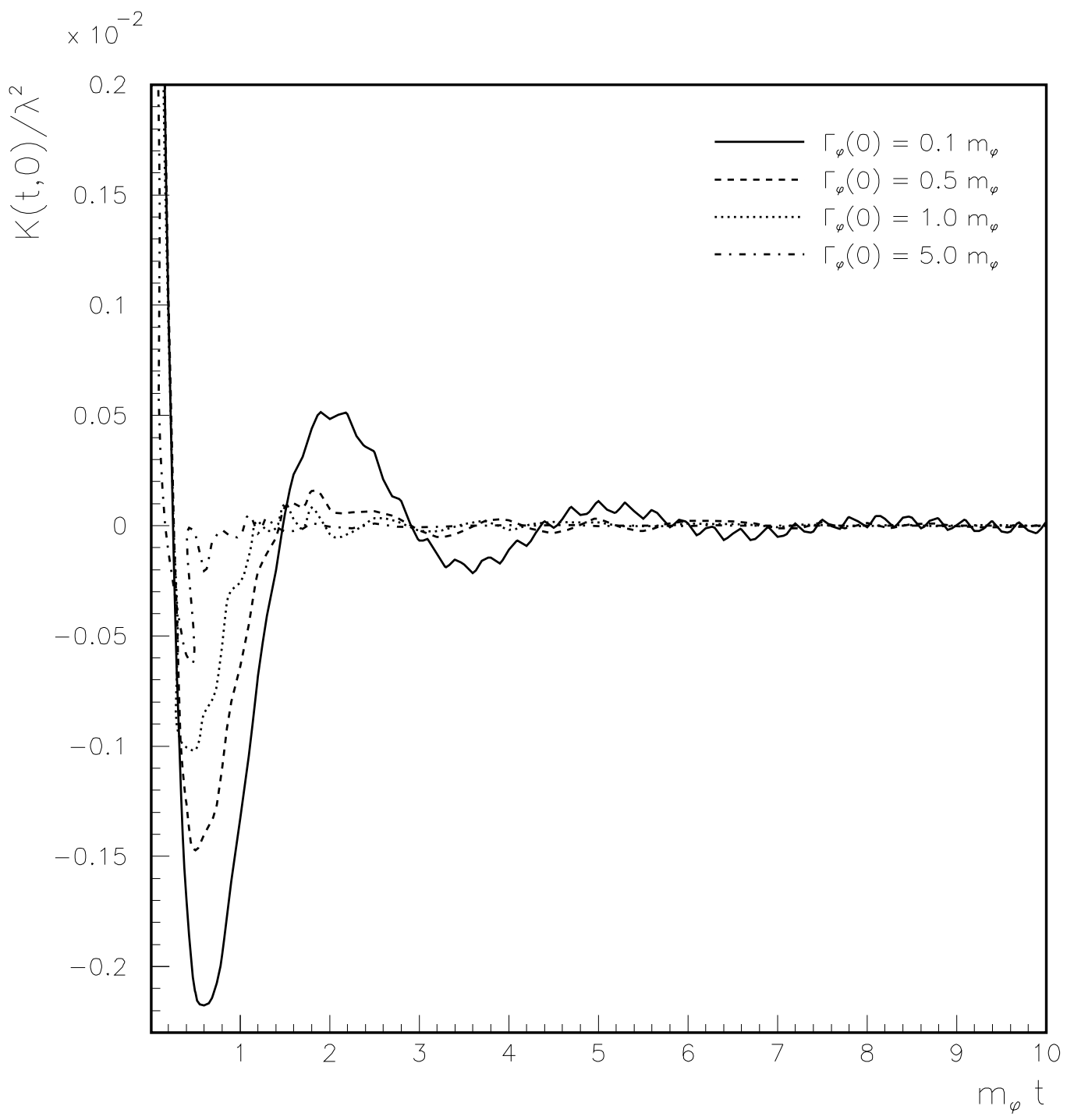




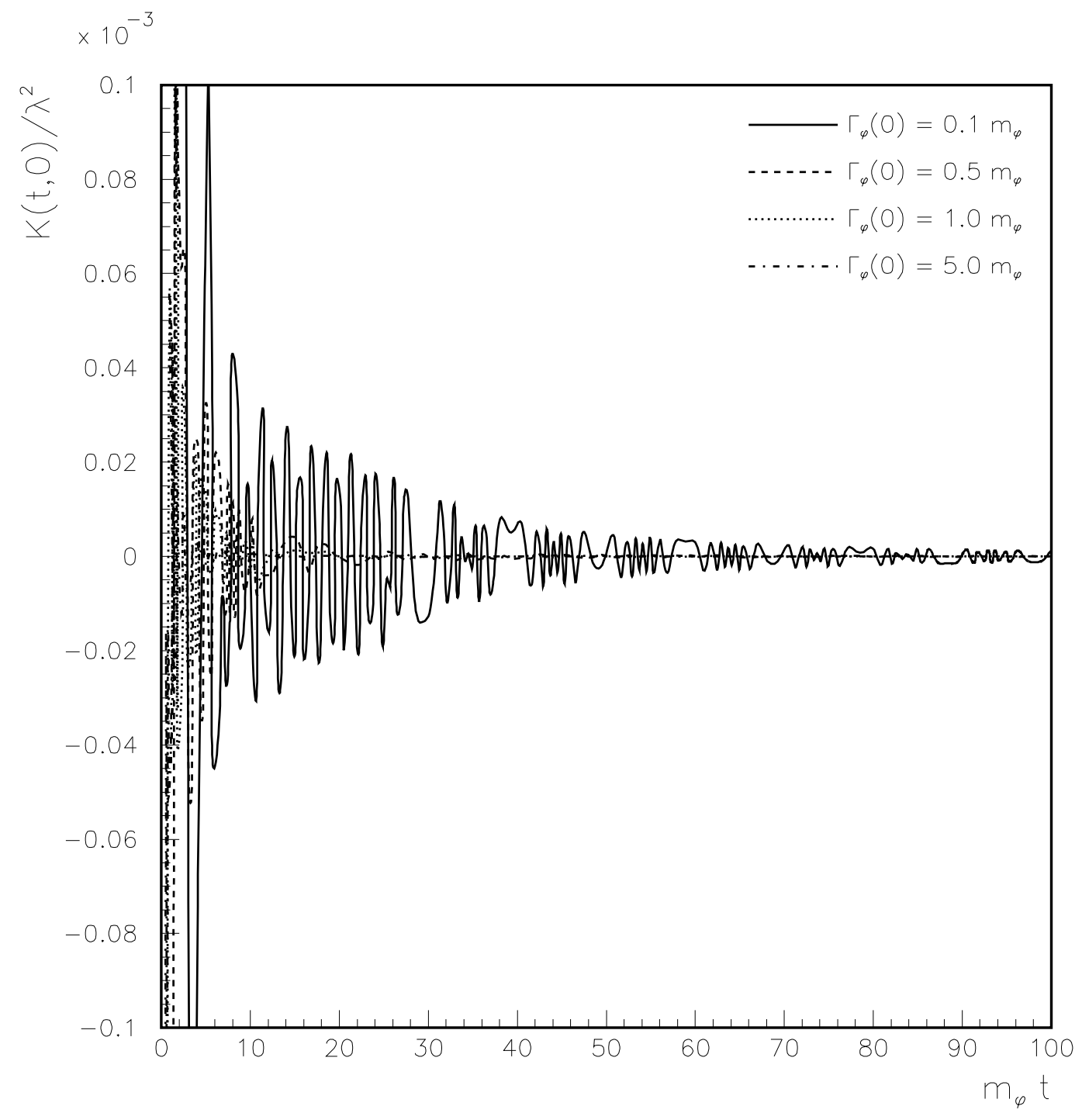

